\title{
An electron transfer path connects subunits of a mycobacterial respiratory supercomplex
}

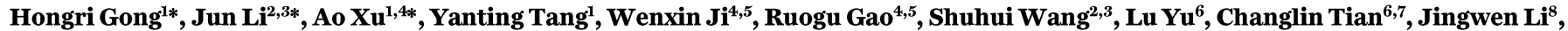
Hsin-Yung Yen ${ }^{8,9}$, Sin Man Lam ${ }^{10}$, Guanghou Shui' ${ }^{10}$, Xiuna Yang ${ }^{2,3}$, Yuna Sun ${ }^{4}$, Xuemei Li ${ }^{4}$, Minze Jia ${ }^{4}$, Cheng Yang', Biao Jiang ${ }^{2}$, Zhiyong Lou $^{11}$, Carol V. Robinson ${ }^{8}$, Luet-Lok Wong' ${ }^{12}$, Luke W. Guddat ${ }^{13}$, Fei Sun ${ }^{4,5}+$, Quan Wang ${ }^{4}+$, Zihe Rao ${ }^{1,2,3,4,11}$ †

${ }^{1}$ State Key Laboratory of Medicinal Chemical Biology and College of Life Science, Nankai University, Tianjin 300353, China. ${ }^{2}$ Shanghai Institute for Advanced Immunochemical Studies, ShanghaiTech University, Shanghai, 201210, China. ${ }^{3} \mathrm{CAS}$ Center for Excellence in Molecular Cell Science, Shanghai Institute of Biochemistry and Cell Biology, Chinese Academy of Sciences, 320 Yueyang Road, Shanghai 200031, China. ${ }^{4}$ National Laboratory of Biomacromolecules, CAS Center for Excellence in Biomacromolecules, Institute of Biophysics, Chinese Academy of Sciences, Beijing 100101, China. ${ }^{5}$ University of Chinese Academy of Sciences, Beijing, China. ${ }^{6} \mathrm{High}$ Magnetic Field Laboratory, Chinese Academy of Sciences, Hefei 230031, China. ${ }^{7}$ Hefei National Laboratory of Physical Sciences at Microscale and School of Life Sciences, University of Science and Technology of China, Hefei 230027, China. ${ }^{8}$ Department of Chemistry, University of Oxford, Physical and Theoretical Chemistry Laboratory, South Parks Rd, Oxford, OX13QZ, UK. ${ }^{9}$ OMass Technologies Ltd., Begbroke Science Park, Woodstock Rd, Yarnton, Kidlington OX5 1PF, UK. ${ }^{10}$ State Key Laboratory of Molecular Developmental Biology, Institute of Genetics and Developmental Biology, Chinese Academy of Sciences, Beijing 100101, China. ${ }^{11}$ Laboratory of Structural Biology, Tsinghua University, Beijing 100084, China. ${ }^{12}$ Department of Chemistry, University of Oxford, Inorganic Chemistry Laboratory, South Parks Road, Oxford OX1 3QR, UK. ${ }^{13}$ School of Chemistry and Molecular Biosciences, The University of Queensland, Brisbane, 4072 Queensland, Australia.
\end{abstract}

*These authors contributed equally to this work.

†Corresponding authors. Email: raozh@tsinghua.edu.cn (Z.R.); wangq@ibp.ac.cn (Q.W.); feisun@ibp.ac.cn (F.S.)

We report a 3.5- $\AA$ resolution cryo-EM structure of a respiratory supercomplex isolated from Mycobacterium smegmatis. It comprises a complex III dimer flanked on either side by individual complex IV subunits. Complex III and IV associate such that electrons can be transferred from quinol in complex III to the oxygen reduction center in complex IV via a bridging cytochrome subunit. We observe a superoxide dismutase-like subunit at the periplasmic face, which may be responsible for detoxification of superoxide formed by complex III. The structure reveals features of an established drug target and provides a foundation for development of treatments for human tuberculosis.

In cellular respiration, chemical energy is extracted by coupling the oxidation of an energy source (e.g., sugars, fatty acids, amino acids) and the reduction of an electron acceptor (e.g., oxygen, sulfur, nitrate, sulfate) to synthesize adenosine triphosphate (ATP) which powers cellular reactions. In aerobic organisms, electrons are transferred from electron donors to oxygen, the terminal acceptor, through the electron transport chain (ETC) to pump protons across a membrane (cellular or mitochondrial). This creates a transmembrane proton gradient (proton motive force, PMF) that drives ATP synthesis (1). In ETCs, quinones and cytochromes are two types of electron carriers that shuttle electrons to and from large macromolecular structures embedded in the membrane. In the mitochondrial respiratory chain, four membrane oxidoreductases are involved in electron transfer: complex I (NADH:ubiquinone oxidoreductase, CI), complex II (succinate:ubiquinone oxidoreductase, CII), complex III ( $b c_{1}$-type ubiquinol:cytochrome $c$ oxidoreductase, $b c_{1}$-type CIII) and complex IV ( $a a_{3}$-type cytochrome $c$ oxidase, $a a_{3^{-}}$ type CIV). CIII oxidizes ubiquinol to ubiquinone and passes the electrons to soluble cytochrome $c$ which then shuttles them to CIV, where oxygen is reduced to water (Fig. 1A). The transmembrane PMF is generated by proton pumping in $\mathrm{CI}$, CIII and CIV.

The situation is more complicated in prokaryotic respiratory chains (Fig. 1A) (2). A soluble cytochrome $c$ pathway similar to that in mitochondria is common in Gram-negative bacteria. Variations include a membrane-anchored cytochrome $c$ mediating electron transfer from CIII to CIV (3) and a $c a a_{3}$-type CIV with a fused cytochrome $c$ domain (4). Grampositive bacteria usually possess cytochrome $c$ proteins that are anchored onto the membrane (5), or a fused cytochrome $c$ domain (bcc-type CIII or $c a a_{3}$-type CIV) to mediate electron transfer between CIII and CIV $(6,7)$. Mycobacteria and other Actinobacteria such as Corynebacterium glutamicum are inherently devoid of a soluble cytochrome $c$ in their genomes (8), but contain a $b c c$-type CIII with a di-heme cytochrome $c$ domain fused to CIII $(9,10)$. Variations are also observed within CIII and CIV. Alternative Complex IIIs (ACIIIs) which are structurally unrelated to $b c_{1}$-type CIII mediate quinol oxidation (11). Quinol oxidases couple quinol oxidation to oxygen reduction without the need for cytochrome $c$ (12). Alternative oxidases (Aox) catalyze quinol oxidation/oxygen reduction without proton pumping (13). 
Despite variation with ETCs, homologs or analogs of CI through to CIV are the most common components of respiratory chains in aerobic organisms. Structures of both prokaryotic and eukaryotic CI (14-16), CII $(17,18)$, CIII (19-21) and CIV (22-24) have been determined, elucidating the flow of electrons within these individual complexes. Structural information for the mitochondrial respirasome $\mathrm{CI}_{1} \mathrm{CIII}_{2} \mathrm{CIV}_{1}$ and preliminary electron microscopic study of a $\mathrm{CIII}_{2} \mathrm{CIV}_{2}$ supercomplex from yeast have been reported $(25,26)$. However, cytochrome $c$ is not visible in any of these structures. Thus, a complete pathway for electron flow is yet to be fully elucidated. Understanding the precise details of the structural assembly for a CIII-CIV supercomplex will greatly assist in this endeavor $(27,28)$. It has been reported recently that respiratory supercomplexes in situ have a conserved core of CI and a dimer of CIII but otherwise their stoichiometry and structure vary (29). Up to two copies of monomeric CIV were found associated with the $\mathrm{CI}_{1} \mathrm{CIII}_{2}$ assembly in bovine heart and the yeast Yarrowia lipolytica, but their positions varied (30). The conserved features of supercomplex assemblies such as $\mathrm{CI}_{1} \mathrm{CIII}_{2}$ and $\mathrm{CIII}_{2} \mathrm{CIV}_{2}$ suggest that these types of associations have important roles in respiratory electron transfer.

The $b c c$-type CIII from Actinobacteria has a di-heme $c$ subunit (7). It has been suggested that one cytochrome $c$ domain is the donor for the $a a_{3}$-type CIV and the other is the acceptor for the CIII Rieske Fe-S protein (31). In support of this concept of intra-supercomplex electron transfer, CIII-CIV supercomplexes have been isolated from $M$. smegmatis, $C$. glutamicum, and M. tuberculosis and shown to couple quinol oxidation to oxygen reduction without an external electron shuttle $(9,10,32)$. Therefore, structural data for the $b c c-a a_{3}$ type CIII-CIV supercomplex (SC III-IV) can provide answers as to how CIII and CIV are coupled and how electrons are transferred from CIII to CIV.

\section{Purification and characterization of SC III-IV}

To isolate the SC III-IV in a functional form, we engineered the genome of M. smegmatis to incorporate a $10 \times$ His tag at the C terminus of the QcrB subunit of CIII, extracted and purified the CIII-CIV complex by Ni-NTA affinity chromatography and gel filtration. Gel filtration and Blue-Native PAGE showed a single peak and a single band, suggesting a highly-ordered supramolecular assembly (fig. S1, A and B). SDS-PAGE and mass spectrometry confirmed the presence of all the known components of CIII and CIV as well as several previously unknown components (fig. S1D and table S1). Native Orbitrap mass spectrometry gave a molecular weight of 873.4 $\mathrm{kDa} \pm 10.4 \mathrm{Da}$ for the complex (fig. S1E). Both the electronic absorption spectrum and the electron paramagnetic resonance (EPR) spectrum showed peaks expected from the various hemes, copper centers and [2Fe-2S] prosthetic groups in CIII and CIV (fig. S1K). Since the $b c c: a a_{3}$ preparations from $M$. smegmatis are active with the more soluble menadiol (2methyl-1,4-naphthoquinol) as substrate (33), the quinol:oxygen oxidoreductase activity of SC III-IV was assayed by measuring the rate of $\mathrm{O}_{2}$ consumption in the presence of menadiol. SC III-IV oxidized menadiol and reduced $\mathrm{O}_{2}$ with an apparent $k_{\text {cat }}$ of $2.80 \pm 0.05 \mathrm{~s}^{-1}$ for $\mathrm{O}_{2}$ consumption or $11.20 \pm 0.20$ $\mathrm{e}^{-} \mathrm{s}^{-1}$ and a $K_{\mathrm{m}}$ of $120.70 \pm 3.72 \mu \mathrm{M}$ for menadiol (fig. S2, A, B, and C). The $k_{\text {cat }}$ value is comparable to the $65 \mathrm{e}^{-} \mathrm{s}^{-1}$ reported for this complex with 2,3-dimethyl-1,4-naphthoquinol $\left(\mathrm{DMNQH}_{2}\right)$ as the electron donor (9); the difference is likely due to $\mathrm{DMNQH}_{2}$ being more reducing and a closer structural analog of the natural substrate menaquinol than menadiol. The data confirm that the purified sample is a functioning supercomplex containing CIII and CIV and capable of directly coupling quinol oxidation to oxygen reduction.

\section{Overall architecture of SC III-IV}

The structure of SC III-IV was determined by cryo-EM to an overall resolution of $3.5 \AA$ (Fig. 1B; table S2; fig. S3, A to $\mathrm{G}$; and movie S1). The dimensions of the supercomplex are $200 \times 70 \times 120 \AA$, with a linear dimeric $\mathrm{CIV}_{1}-\mathrm{CIII}_{2}-\mathrm{CIV}_{1}$ arrangement where individual CIVs flank the central CIII dimer on either side (Fig. 1, B and C, and Movie 1). This $\mathrm{C}_{2}$ symmetrized linear architecture is completely different from those previously reported for respiratory supercomplexes (fig. S4). CIII is comprised of canonical three subunits as a homodimer (Fig. 2A and fig. S5A). In addition to the four known subunits of $M$. smegmatis CIV, two subunits were observed that match two of the newly identified proteins, CtaI and CtaJ (Fig. 2B and fig. S3H), showing a similar topology and binding schema to subunit Va and IV in mitochondrial CIV (fig. S5J).

There is extra density within the interface between CIII and CIV as well as at the top of the CIII dimer (Fig. 1B). The density within the interface could be modeled by two proteins, LpqE and PRSAF1 (prokaryotic respiratory supercomplex association factor 1) (Fig. 1C and Movie 1). LpqE was found to be a N-terminal triacylated lipoprotein (Fig. 4F), with a $N$-acylated-S-diacylated modification of Cys24 within the lipobox $\left({ }^{21} \mathrm{Lxx}^{24} \mathrm{C}-\right)$ sequence (34). On the periplasmic side the density on top of the CIII dimer consisted of a peptide fragment linked with a region of bulk density which could be visualized in a low-pass filtered map. The peptide fragment was modeled with residues Cys21-Pro45 of the Nterminal sequence of superoxide dismutase SodC of $M$. smegmatis. The EM map indicated side chain modifications, including triacylation at Cys21 that was part of a lipobox (${ }^{18} \mathrm{Lxx}^{21} \mathrm{C}-$ ) sequence (Fig. 4E) and possible glycosylation sites (fig. S3H). Native mass spectrometry further identified that SodC is a component of SC III-IV (fig. S1F). The stoichiometry of SodC was confirmed as a dimer through the collisional 
dissociation of the SodC complex. Extensive glycosylation and copper-binding of SodC was observed in the mass spectrum (fig. S1F). The bulk density was therefore fitted with a dimer of SodC (Fig. 1, B and C, and fig. S3F). SC III-IV possesses superoxide dismutase activity, with a specific activity of $132.56 \pm 12.57 \mathrm{IU} / \mathrm{mg}$-SOD, assuming 100\% occupancy (fig. S2E). SodC association with SC III-IV was also confirmed by isolation of the supercomplex by Ni-affinity chromatography when a His-tag was introduced only to the $\mathrm{C}$ terminus of SodC and not any of the CIII and CIV subunits. The SodCtagged form of the complex showed a higher specific activity of $957.36 \pm 23.34 \mathrm{IU} / \mathrm{mg}$-SOD but still lower than the 1000$6000 \mathrm{IU} / \mathrm{mg}$-SOD typically observed with soluble SOD enzymes. It also indicates an $14 \%$ (SodC) $)_{2}$ occupancy in the purified QcrB-tagged SC III-IV particles for cryo-EM study. Thus, it appears that there might be some dissociation of SodC from SC III-IV during detergent solubilization. It is also possible that the SOD occupancy is growth regulated since an up-regulation of sodC (the gene encoding the SOD here) in response to phagocytosis by human macrophages has been reported (35). Further work on the role of SOD in association with SC III-IV is in progress.

All the prosthetic groups predicted from the canonical CIII and CIV subunits were clearly resolved and found to be coordinated with conserved canonical residues (figs. S3J and S8). The menaquinone (MK) molecules were observed at the quinone binding sites in CIII (fig. S7, A and B). The calculated molar ratio between iron atoms and copper atoms in the final model is 1.6, in excellent agreement with the value of 1.5 determined by atomic absorption spectroscopy (fig. S1C). SodC from $M . t b$ does not contain zinc (36), and atomic absorption spectroscopy analysis showed that zinc was absent from SC III-IV (fig. S1C). We were in total able to build 34 phospholipids (fig. S3I) and ten MK molecules (fig. S7) in SC III-IV. The total molecular mass of the model, including the new identified subunits, is $\sim 760 \mathrm{kDa}$ which is lower than the 873.4 $\mathrm{kDa} \pm 10.4 \mathrm{Da}$ determined by native Orbitrap mass spectrometry (fig. S1E). This difference may be accountable on the basis of contributions from the detergents/lipids and the possibility of the presence of additional unidentified subunits.

\section{Structure of CIII and CIV in the supercomplex}

The cryo-EM map clearly shows QcrA, QcrB and QcrC of CIII in a dimeric form with all their prosthetic groups visualized (Fig. 2A; fig. S3, H and J; and Movie 2). QcrA has a "U" shaped structure within its N-terminal domain while the equivalent subunit in $b c_{1}$-type CIII has only one TMH (equiv-

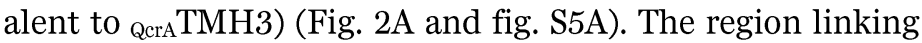
the two arms is located near the cytoplasmic side. The C-terminal domain of ${ }_{\text {Qcra }} \mathrm{TMH} 3$ is on the periplasmic side and holds the [2Fe-2S] cluster. Importantly, QcrA here also has a roof-like structure on the periplasmic side which is involved in the dimerization of CIII (Fig. 2A) while the $b c_{1}$-type CIIIs do not have this feature. The heme $b_{\mathrm{H}}$ and heme $b_{\mathrm{L}}$ cofactors are bound within four TMHs in QcrB (Fig. 2A). The N-terminal periplasmic portion of QcrC can be divided into two heme-containing cytochrome $c$ domains D1 and D2 (equivalent to the $c_{1}$ domain in $b c_{1}$ complex) (Fig. $2 \mathrm{~A}$ and fig. $\mathrm{S} 5 \mathrm{~N}$ ). These two domains are in close contact with and face each other in an anti-parallel orientation. The D2 domain interacts extensively with QcrA and QcrB, while the additional D1 domain protrudes out of the core of CIII and is involved in direct intra-supercomplex electron transfer (see below). Overall, although the $b c c$-type CIII in the supercomplex shares a similar dimeric association as the bacterial $b c_{1}$-type CIII and mitochondrial CIII, the structural details are markedly different (fig. S5B).

CIV in SC III-IV belongs to the type A heme-copper oxidase (HCO) family (Fig. 2B and Movie 3) (37). The central cavity of the barrel-like arrangement of the 12 TMHs in the CtaD subunit holds heme $a$, heme $a_{3}$ and $\mathrm{Cu}_{\mathrm{B}}$. The C-terminal hydrophilic $\beta$ barrel domain of $\mathrm{CtaC}$ holds the two $\mathrm{Cu}_{\mathrm{A}}$ ions. The four protons required for oxygen reduction by heme $a_{3}: \mathrm{Cu}_{\mathrm{B}}$ in CIVs are transferred to the catalytic center through two pathways denoted D and K (38). Due to the limited resolution, water molecules are not observed in our model. However, structure comparison revealed that the $\mathrm{D}$ and $\mathrm{K}$ pathways are conserved in SC III-IV (fig. S5, K and L).

\section{Interaction between CIII and CIV and the contribu- tion of association subunits and lipids}

The linear form of SC III-IV arises from the dimerization of CIII which is mediated by contacts between subunits QcrA and QcrB (Fig. 2A and fig. S6, A to D). There are extensive contacts between CIII and CIV on both the cytoplasmic and periplasmic sides of the membrane (Fig. 3). Of the three association subunits LpqE, PRSAF1 and SOD in SC III-IV, both LpqE and PRSAF1 form numerous contacts with CIII and CIV, suggesting an important role for both in supercomplex stability (Fig. 3B and fig. S6, E to G). SOD utilizes its lipidmodified N-terminal fragment to associate with CIII (Fig. 4E) and forms a dimer on the periplasmic side of the CIII dimer. There are no direct interactions between the main body of the SOD dimer and CIII or CIV. This might allow a flexible orientation for this subunit which could be important for efficient clearance of ROS generated by side reactions when electrons are transferred from CIII to CIV (see below).

Phospholipids, especially cardiolipin (CL), are known to contribute to both the assembly and stability of respiratory complexes and supercomplexes (39). In the structure of SC III-IV, phospholipids are identified in the transmembrane space of CIII and CIV and the interface between CIII and CIV (Fig. 4). Of particular note are the four CL molecules in the 
large groove between CIII and CIV (Fig. 4D); the sixteen fatty acid chains fill most of the space in the groove and may play an important role in stabilizing the supercomplex. The N-terminal modified lipid tails of the lipoproteins SOD and LpqE mediate inter-subunit hydrophobic interactions and contribute to the stability of the supercomplex (Fig. 4, E and F). These lipid modifications described here are similar to those observed in a recently described structure of alternative complex III (11).

\section{Quinone and quinone binding pockets}

Quinone binding sites of respiratory complexes are of great interest because they are part of the Q-cycle hypothesis. They have varied sequences and specificities between species, and are often the sites for inhibitor binding and thus are important for drug discovery. We have identified the two quinone binding sites $\left(Q_{P}\right.$ and $\left.Q_{V}\right)$ in SC III-IV (Fig. 5). The quinol oxidation site $\left(Q_{P}\right.$ site) responsible for menaquinol $\left(\mathrm{MKH}_{2}\right)$ oxidation is near heme $b_{\mathrm{L}}$ while the quinone reduction site $\left(\mathrm{Q}_{\mathrm{N}}\right.$ site) responsible for menaquinone (MK) reduction is close to heme $b_{\mathrm{H}}$.

The $Q_{P}$ site near heme $b_{\mathrm{L}}$ is at the center of an inverted triangle structure and surrounded by helices (Fig. 5A). Residues at this site are not conserved compared to the $b c_{1}$ complex (Fig. 5C). The typical "PEWY" motif in the $b c_{1}$ complex is replaced by "PDFY" (Fig. 5C). One MK molecule is identified at this site with its naphthoquinone ring surrounded mainly by hydrophobic residues. The edge-to-edge distance from MK to heme $b_{\mathrm{L}}$ is $16 \AA$. Thus, we speculate that the endogenous electron donor $\mathrm{MKH}_{2}$ would bind closer to heme $b_{\mathrm{L}}$ to facilitate electron transfer and what we observe here might be a representation of the oxidized product as it leaves the $Q_{p}$ site. Furthermore, there are no observed hydrogen bonds to the carbonyl groups of MK (Fig. 5A). It is known that proton abstraction from $\mathrm{MKH}_{2}$ is coupled to electron donation to CIII. Thus, hydrogen bonds are needed between the binding residues and the hydroxyl group of $\mathrm{MKH}_{2}$ to help deprotonate the substrate. Hence $\mathrm{MKH}_{2}$ should bind deeper inside the pocket, close to polar residues such as ${ }_{\text {QcrB }}$ Tyr159, ${ }_{\text {QcrB }}$ Thr308, Qcr3Asp309 (Fig. 5A). Structural superposition with the inhibitor bound $b c_{1}$ complex (40) shows that the MK at the $Q_{P}$ site binds deeper into the pocket in the $b c_{1}$ complex than in this SC III-IV complex.

By contrast, the head group of MK at the $\mathrm{Q}_{\mathrm{N}}$ site is bound in a similar fashion to ubiquinone in the structures of $b c_{1}$ complexes (Fig. 5B). As found for the $Q_{p}$ site, these residues are not conserved compared to the $b c_{1}$ complexes from bacteria to eukaryotes (Fig. 5C). In particular, the carbonyl groups of ubiquinone are coordinated by the conserved His and Asp amongst $b c_{1}$ complexes; these residues are substituted by

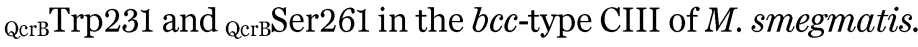
The carbonyl groups of MK in SC III-IV interact with the side chains of ${ }_{\text {QcrB }}$ Tyr48 and ${ }_{\text {QcrBSer261 }}$ that may supply protons for MK reduction. The MK head group is ideally placed for electron transfer, being within $5 \AA$ of the A-edge of heme $b_{\mathrm{H}}$.

Besides finding quinone molecules at the $\mathrm{Q}$ sites, we also observed map signals for another three possible $\mathrm{MK} / \mathrm{MKH}_{2}$ molecules in the supercomplex (fig. S7, C to E). However, further experiments are needed to clarify the identity and function of these molecules.

The new family of candidate anti-mycobacterials, the imidazo[1,2-a]pyridines (IP) represented by Q203 operate by competing with menaquinone for binding at the $Q_{P}$ site of CIII of $M$. tuberculosis (41). Sequence alignments indicate a high similarity between the $Q_{\mathrm{P}}$ sites of CIIIs from $M$. tuberculosis and M. smegmatis (Fig. 5C), thus suggesting Q203 would also have a similar binding mechanism and a similar effect on the activity of $M$. smegmatis CIII. Indeed, recent studies of the anti-mycobacterial activity of Q203 on M. tuberculosis and M. smegmatis demonstrated that Q203 targets the bcc complex in both with similar affinity (42). We investigated the in vitro inhibition of $M$. smegmatis SC III-IV by Q203 by the menadiol/oxygen oxidoreductase activity assay and compared the effect with a hybrid supercomplex of $M$. tuberculosis $b c c$-CIII and $M$. smegmatis $a a_{3}$-CIV. Q203 showed inhibition of menadiol-induced oxygen consumption with $\mathrm{IC}_{50}$ values of $0.84 \pm 0.22 \mu \mathrm{M}$ and $0.61 \pm 0.16 \mu \mathrm{M}$ for SC IIIIV and the hybrid supercomplex, respectively (fig. S2D).

\section{Prosthetic groups and implication for direct electron transfer}

The prosthetic groups of CIII (heme $b_{\mathrm{H}} / b_{\mathrm{L}},[2 \mathrm{Fe}-2 \mathrm{~S}]$ clusters and heme $\left.c_{\mathrm{D} 1} / c_{\mathrm{D} 2}\right)$ and $\mathrm{CIV}\left(\mathrm{Cu}_{\mathrm{A}}, \mathrm{Cu}_{\mathrm{B}}\right.$ and heme $\left.a / a_{3}\right)$ are clearly identified from the cryo-EM map (Fig. 2, A and B, and fig. S3J). The redox centers in CIII and CIV are within distances that allow long-range electron transfer (Fig. 6A). Both heme $b_{\mathrm{L}}$ and heme $b_{\mathrm{H}}$ are found in $\mathrm{QcrB}_{\mathrm{CIII}}$ (figs. S3J and S8A). The edge-to-edge distance between the two heme groups is 12 $\AA$, allowing rapid inter-heme electron transfer. The shortest distance from $b_{\mathrm{L}}$ to $\mathrm{MK}$ at the $\mathrm{Q}_{\mathrm{P}}$ site and from $b_{\mathrm{H}}$ to $\mathrm{MK}$ at the $Q_{\mathrm{N}}$ site are $16 \AA$ and $5 \AA$, respectively (Fig. $6 \mathrm{~A}$ ). Previous studies have proposed that CIIIs from different species adopt a dimeric architecture and form a $\mathrm{H}$-shaped electron transfer system that distributes electrons between four quinone oxidation-reduction sites within the CIII dimer (43). Consistent with this hypothesis, upon dimerization of M. smegmatis CIII, the two $b_{\mathrm{L}}$ heme groups from the CIII monomers are 14 $\AA$ apart (Fig. 6A), allowing electron tunneling between the two hemes.

The heme $c_{\mathrm{D} 1}$-containing D1 domain of $\mathrm{QcrC}_{\mathrm{CII}}$ protrudes into the periplasm and interacts with the $\mathrm{Cu}_{\mathrm{A}}$-containing periplasmic domain of $\mathrm{CtaC}_{\mathrm{CIV}}$ (Fig. 6A). The edge-to-edge distance from heme $c_{\mathrm{D} 1}$ to the $\mathrm{Cu}_{\mathrm{A}}$ center is $12 \mathrm{~A}^{\circ}$. At the interface between these two domains there is a gating residue 
CtacTrp138 on the $\mathrm{Cu}_{\mathrm{A}}$ binding loop. The distance between

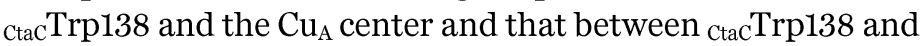
heme $c_{\mathrm{D} 1}$ are approximately equal. It has been proposed, based on mutagenesis studies, that the corresponding Trp121 (44) of $P$. denitrificans CIV is the electron entry site from cytochrome $c$. CIVs from $B$. taurus also possesses a tryptophan residue at the equivalent location (45). Thus, the heme $c_{\mathrm{DI}^{-}}$ containing D1 domain of $\mathrm{QcrC}_{\mathrm{CIII}}$ here interacts with CIV on a similar electron entry site as in other respiratory complexes.

Within CIV, the heme $a$ group is just below the $\mathrm{Cu}_{\mathrm{A}}$ center and the heme $a_{3}$ group is beside heme $a$ (Fig. $6 \mathrm{~A}$ ). $\mathrm{Cu}_{\mathrm{B}}$ is coordinated to three conserved His residues. The space between the iron of heme $a_{3}$ and $\mathrm{Cu}_{\mathrm{B}}$ is the catalytic center for reduction of oxygen. In proximity to propionate groups of heme $a_{3}$, a $\mathrm{Cu}_{\mathrm{C}}$ ion was modeled according to the cryo-EM map (fig. S3J). In other reported CIV structures the equivalent density at this site is occupied by a water molecule (46) or a $\mathrm{Mg}^{2+}$ ion (47). However, we did not detect magnesium in our AAS analysis (fig. S1C). The edge-to-edge distance between $\mathrm{Cu}_{\mathrm{C}}$ and the $\mathrm{Cu}_{\mathrm{A}}$ center and that between $\mathrm{Cu}_{\mathrm{C}}$ and heme $a_{3}$ are $12 \AA$ and 9 $\AA$, respectively.

From the positions of the prosthetic groups in $M$. smegmatis $\mathrm{SC} \mathrm{CIII}_{2} \mathrm{CIV}_{2} \mathrm{SOD}_{2}$ and the redox center separations, it is possible to trace an uninterrupted pathway for the flow of electrons within the supercomplex starting from the electron donor at the $\mathrm{Q}_{\mathrm{P}}$ site in CIII to the final site of oxygen reduction in CIV (Fig. 6B). Menaquinol $\left(\mathrm{MKH}_{2}\right)$ from the Q-pool binds at the $Q_{p}$ site near heme $b_{\mathrm{L}}$ and transfers one electron to the [2Fe-2S] cluster and the other to heme $b_{\mathrm{L}}$ which passes the electron via heme $b_{\mathrm{H}}$ to a menaquinone (MK) bound at the $\mathrm{Q}_{\mathrm{N}}$ site, generating the highly reactive intermediate menasemiquinone $(\mathrm{MK} \bullet)$. The reduced [2Fe-2S] cluster is within electron tunneling distance to transfer an electron to heme $c_{\mathrm{D} 2}$ which rapidly passes the electron to heme $c_{\mathrm{D} 1}$. A second $\mathrm{MKH}_{2}$ then binds at the $\mathrm{Q}_{\mathrm{P}}$ site and repeats the process. The $\mathrm{MK} \cdot$ at the $\mathrm{Q}_{\mathrm{N}}$ site is fully reduced to $\mathrm{MKH}_{2}$ and released to the Q-pool. This completes the Q cycle. An electron path between CIIIs in the dimer is also possible through tunneling between the adjacent $b_{\mathrm{L}}$ heme groups, albeit with low efficiency (43). The reduced [2Fe-2S] cluster again transfers the other electron to heme $c_{\mathrm{D} 2} / c_{\mathrm{D} 1}$. Once heme $c_{\mathrm{D} 1}$ is reduced, two $\mathrm{Cu}_{\mathrm{A}}$ ions of $\mathrm{CtaC}_{\mathrm{CIV}}$ accept the electron through contacts forged with the $\mathrm{D} 1$ domain of $\mathrm{QcrC}_{\mathrm{CIII}}$. At this point, an electron is transferred from CIII to CIV. Within CIV, the electron is transferred through heme $a$ (or possibly the $\mathrm{Cu}_{\mathrm{C}}$ center) and finally reaches the terminal heme $a_{3}: \mathrm{Cu}_{\mathrm{B}}$ reaction center for oxygen reduction. As a consequence of the electron transfer, protons are translocated to the periplasm, forming a transmembrane PMF. Throughout the entire pathway from the $Q_{p}$ site to the terminal oxygen reduction center, electrons tunnel between prosthetic groups that are all buried inside this integral complex.
Quinone reduction at the $\mathrm{Q}_{\mathrm{N}}$ site to complete the $\mathrm{Q}$ cycle can be bypassed/short-circuited if both electrons from $\mathrm{MKH}_{2}$ oxidation at the $\mathrm{Q}_{\mathrm{P}}$ site are transferred to the [2Fe-2S] cluster and then to CIV for oxygen reduction. Hence, competent energy transduction requires that electron transfer from the $Q_{P}$ site has to be bifurcated between reduction of the [2Fe-2S] cluster and heme $b_{\mathrm{L}}$. In essence, the pathway for transferring electrons from the [2Fe-2S] cluster eventually to CIV has to be sufficiently slow for electron transfer to the $Q_{N}$ site for quinone reduction to occur. In the $b c_{1}$ complex the [2Fe-2S] cluster domain cycles between " $b$ " and " $c_{1}$ " states. In the $b$-state the [2Fe-2S] cluster is close to the $\mathrm{Q}_{\mathrm{P}}$ site to accept an electron from quinol oxidation but too far away (26 ̊) (fig. S5E) to transfer an electron to the $c_{1}$ heme at an appreciable rate. The cluster domain undergoes a "head displacement" conformation change to the $c_{1}$-state where the [2Fe-2S] cluster moves to within $11 \AA$ of the $c_{1}$ heme to facilitate electron transfer. This head displacement or "gating" step occurs with a rate constant of $6 \times 10^{4} \mathrm{~s}^{-1}(48)$.

We cannot rule out the possibility that SC III-IV could adopt a different conformation and cycle between states similar to the $b$ and $c_{1}$ states of $b c_{1}$ complexes. However, the dimer of QcrA is held firmly in place by the periplasmic roof-like structure and further surrounded by QcrB and QcrC, which might limit the space for the potential conformational change (fig. S5D). Structural superposition shows that the position of the [2Fe-2S] in QcrA is similar to that of $b c_{1}$-type CIII in the $b$ state, i.e., rapid cluster reduction by $\mathrm{MKH}_{2}$ can occur. The [2Fe-2S] cluster is at a distance of $16 \AA$ from heme $c_{\mathrm{D} 2}$. We used this distance, the reported midpoint potentials of the prosthetic groups (10), and a reorganization energy $(\lambda)$ of 0.7 $\mathrm{eV}$ to calculate a rate constant of electron transfer of $5.6 \times 10^{2}$ $\mathrm{s}^{-1}$ from [2Fe-2S] to heme $c_{\mathrm{D} 2}$ (49). This is much slower than the head displacement gating step in $b c_{1}$ complexes $\left(6 \times 10^{4}\right.$ $\mathrm{S}^{-1}$ ). We conclude that short-circuiting in SC III-IV is likely to be rate-limited by slow electron transfer between the [2Fe2S] cluster and heme $c$ made possible by positioning of the chain of redox centers, rather than by conformational changes as found in $b c_{1}$ complexes.

\section{Role of SOD association}

In aerobic organisms, the respiratory ETC not only generates the energy needed to fuel biological functions but is also a major source of intracellular ROS that can cause damage to cellular structures and components (50). Complex III is one of the major sites of ROS production (51). While ROS are emerging as important elements in the bacterial response to lethal stress (52), it is well documented that they can disturb respiratory activity via oxidative damage of ETC complexes which are in turn protected by the ROS scavengers, SOD and catalase (53-55). Up-regulation of $M$. tuberculosis sodC (the gene encoding the SOD here) in response to phagocytosis by 
human macrophages has been observed (35). This Mycobacterial $\mathrm{Cu}, \mathrm{Zn}$ superoxide dismutase SodC was identified as a membrane-bound enzyme and proposed to protect specific membrane-associated targets from oxy-radical damage, thus facilitating mycobacterial intracellular growth (35). A null sodC mutant of $M$. tuberculosis was shown to be readily killed by externally generated superoxide and by activated macrophages producing oxidative bursts (56). In this work, we found that catalytically active SodC is an integral part of a respiratory supercomplex $\mathrm{CIII}_{2} \mathrm{CIV}_{2} \mathrm{SOD}_{2}$. SodC could serve to scavenge ROS generated locally or by other ETC complexes, as well as ROS released by the immune response of the host. Its recruitment has the potential to make this critical respiratory machinery a robust system even under the high oxidative stress inside macrophages. Immunoblotting of Caenorhabditis elegans respiratory supercomplexes separated by BN-PAGE showed that mitochondrial superoxide dismutase-2 (mtSOD-2) is associated with the respirasome $\mathrm{CI}-\mathrm{CIII}_{2}-\mathrm{CIV}$, suggesting that the mtSOD might also provide similar local protection against ROS damage (57).

\section{Conclusions}

The cryo-EM structure of a CIII-CIV respiratory supercomplex from $M$. smegmatis has revealed a complete intracomplex electron transfer pathway from quinol oxidation in CIII to oxygen reduction in CIV, a new mechanism for bifurcating electron transfer to ensure completion of the $Q$ cycle for energy transduction, and the association of a superoxide dismutase that can provide protection against oxidative damage by reactive oxygen species. The structure of the quinone binding sites also provides a framework for structure-based anti-mycobacterial drug discovery.

\section{Materials and Methods \\ Bacterial Strain}

A $M$. tuberculosis-like highly hydrogen peroxide-resistant $M$. smegmatis mutant strain, $\mathrm{mc}^{2} 51$ (58), was used in this study. The draft genome sequence data can be obtained through the GenBank accession no. JAJD00000000.1. The code for the $10 \times$ His tag was introduced at the $\mathrm{C}$ terminus of the QcrB or SodC genome loci through homologous recombination. This modification allowed the metal affinity purification step. The presence of the histidine tag was confirmed by PCR and Western blot.

\section{M. smegmatis culture and membrane isolation}

Culturing of the cells and membrane isolation were performed as described in previously published protocols, with some modifications $(9,32)$. The cells were grown in LB media supplemented with hygromycin $(50 \mu \mathrm{g} / \mathrm{mL})$, carbenicillin (20 $\mu \mathrm{g} / \mathrm{mL})$ and Tween $80(1 \mathrm{~mL} / \mathrm{L})$, at $37^{\circ} \mathrm{C}$ with shaking to maintain oxygenation. A total of $10 \times 1 \mathrm{~L}$ bacteria solutions were cultured until the $\mathrm{OD}_{600}$ reached $\sim 1.5$ and then harvested by centrifugation for $30 \mathrm{~min}$ at $4,000 \mathrm{rpm}$. The obtained bacteria were resuspended in buffer A (20 mM MOPS, pH 7.4, 100 mM NaCl, 1 mM EDTA, 1 mM PMSF). Cell lysis was achieved by three passes through a high pressure cell disrupter at $4^{\circ} \mathrm{C}$ and 1,200 bar. The lysate was centrifuged at $14,000 \mathrm{rpm}$ for $10 \mathrm{~min}$ to remove cell debris and non-lysed cells. The resulting supernatant was centrifuged at 36,900 rpm for 1 hour in a Ti45 rotor (Beckman). The membrane pellets were harvested and stored at $-80^{\circ} \mathrm{C}$.

\section{Supercomplex purification}

Membranes were thawed and homogenized in buffer A. Respiratory supercomplexes were extracted from the membrane by $1 \%(w / v)$ digitonin for three hours with slow stirring at $4^{\circ} \mathrm{C}$. Insoluble materials were removed by centrifugation at $18,000 \mathrm{rpm}$ for $30 \mathrm{~min}$ at $4^{\circ} \mathrm{C}$. The supernatant was loaded onto a Ni-NTA column followed by gravity feeding three column volumes of buffer A through the resin. The resin was further washed by buffer B (20 mM MOPS, pH 7.4, $100 \mathrm{mM}$ $\mathrm{NaCl}, 1 \mathrm{mM}$ EDTA, $0.1 \%$ (w/v) digitonin, $50 \mathrm{mM}$ imidazole). The protein was eluted with buffer C (20 mM MOPS, pH 7.4, $100 \mathrm{mM} \mathrm{NaCl}, 1 \mathrm{mM}$ EDTA, 0.1\% (v/w) digitonin, $500 \mathrm{mM}$ imidazole). Eluted protein was concentrated using a $100-\mathrm{kDa}$ cut-off centrifugal concentrator (Millipore) and this sample was then loaded onto a Superose 6 (10/300 GL, GE Healthcare) column equilibrated in a buffer containing 20 $\mathrm{mM}$ MOPS, pH 7.4, $100 \mathrm{mM} \mathrm{NaCl}, 1 \mathrm{mM}$ EDTA and $0.1 \%(v / w)$ digitonin. The peak fractions (elution volume between 12.25 $\mathrm{mL}$ and $13 \mathrm{~mL}$ ) were pooled and concentrated to $5.5 \mathrm{mg} \mathrm{mL}^{-1}$ by using a $100-\mathrm{kDa}$ cutoff centrifugal concentrator.

\section{Characterization of the respiratory supercomplex $\mathrm{CHI}_{2} \mathrm{CIV}_{2} \mathrm{SOD}_{2}$}

The supercomplex was characterized by optical spectroscopy, mass spectrometry (MS) and 3,3'-diaminobenzidine (DAB) staining. To identify the heme groups, selected fractions were analyzed by recording spectra from 250 to $700 \mathrm{~nm}$ before and after reduction with dithionite according to previously described methods $(9,32)$. To detect the protein components, the concentrated supercomplex sample was subjected to MS analysis at the National Center for Protein Science (Shanghai, China). The protein sample was analyzed by Blue Native PAGE (BN-PAGE) and then a separate strip of the gel was stained with DAB (59-61).

\section{Pyridine haemochrome assay}

For denaturing redox difference spectra samples were taken up in $20 \%(\mathrm{v} / \mathrm{v})$ pyridine, $0.1 \mathrm{M} \mathrm{NaOH}$. Redox difference spectra were recorded in the range 500-620 nm using potassium ferricyanide for oxidation and sodium dithionite for reduction. Potassium ferricyanide $(250 \mu \mathrm{M})$ was added to the 
sample and the spectrum of this oxidized form was recorded. Sodium dithionite $(5 \mathrm{mM})$ was then added to this sample, the solution was mixed well and the spectrum was scanned repeatedly until it remained unchanged, giving the spectrum of the reduced form. The heme $a, b$ and $c$ contents were determined from the reduced-minus-oxidized difference spectrum using the following extinction coefficients $\Delta \varepsilon_{587-620}=21.7 \mathrm{mM}^{-}$ ${ }^{1} \cdot \mathrm{cm}^{-1}, \Delta \varepsilon_{557-540}=23.98 \mathrm{mM}^{-1} \cdot \mathrm{cm}^{-1}, \Delta \varepsilon_{550-535}=23.97 \mathrm{mM}^{-1} \cdot \mathrm{cm}^{-1}$.

\section{Native mass spectrometry of supercomplex}

Purified supercomplex was buffer exchanged into 200 $\mathrm{mM}$ ammonium acetate buffer $\mathrm{pH} 7.5$ containing DDM at 2 times critical micelle concentration. The sample was immediately introduced into a modified Q-Exactive mass spectrometer (Thermo) and ions were transferred into the Higher-energy collisional dissociation (HCD) cell following a gentle voltage gradient (injection flatapole, inter-flatapole lens, bent flatapole, transfer multipole: $7.9,6.94,5.9,4 \mathrm{~V}$ respectively). The capillary voltage was maintained at 1.2-1.4 eV at temperature of $200^{\circ} \mathrm{C}$. The optimized acceleration voltage for intact supercomplex is $150 \mathrm{~V}$ and $100-120 \mathrm{~V}$ in the source and HCD cell, respectively. SodC subunits were dissociated from the complex with high acceleration voltage (250-300 V) in the source and the collisional dissociation of dimeric SodC was performed with the voltage ramp from $0-150 \mathrm{~V}$ in HCD cells. Backing pressure was maintained at $\sim 1.20 \times 10^{-9}$ mbar and data was analyzed using Xcalibur 2.2 SP1.48.

\section{Independent lipidomics analysis 1}

Co-purified lipids from supercomplex were extracted by chloroform/methanol $(2: 1, \mathrm{v} / \mathrm{v})$ and lyophilized and re-dissolved in $60 \%$ acetonitrile (ACN). For LC-MS/MS analysis, the extracted lipids were loaded into a $5-\mu \mathrm{l}$ sample loop using an autosampler by a full-loop method with an overfill factor of 1.4, and then transferred to a $1-\mu l$ injection loop using a loading pump at a flow rate of $5 \mu \mathrm{l} / \mathrm{min}$ with $70 \%$ solution A and $30 \%$ solution $\mathrm{B}$. The lipids in the injection loop were injected onto a C18 column (Acclaim PepMap 100, C18, $75 \mu \mathrm{m} \times 15 \mathrm{~cm}$, Thermo Fisher Scientific) using a nanopump at a flow rate of $300 \mathrm{nl} / \mathrm{min}, 30 \%$ solution B. The lipids were separated on the C18 column at $40^{\circ} \mathrm{C}$ by a gradient starting from $30 \%$ solution B. After $10 \mathrm{~min}$, solution B was ramped to $65 \%$ over $1 \mathrm{~min}$, then $80 \%$ over $6 \mathrm{~min}$, before being held at $80 \%$ for $10 \mathrm{~min}$, then ramped to $99 \%$ over $6 \mathrm{~min}$ and held for $7 \mathrm{~min}$. (Solution A: (ACN: $\mathrm{H} 2 \mathrm{O}$ (60:40), $10 \mathrm{mM}$ ammonium formate, $0.1 \%$ formic acid) and solution B (IPA: ACN (90:10), $10 \mathrm{mM}$ ammonium formate, $0,1 \%$ formic acid)). The column eluent was delivered via a dynamic nanospray source to a hybrid LTQ Orbitrap mass spectrometer (Thermo Scientific). Typical MS conditions were: spray voltage $(1.8 \mathrm{kV})$ and capillary temperature $\left(175^{\circ} \mathrm{C}\right)$. The LTQ-Orbitrap XL was operated in negative ion mode and in data-dependent acquisition with one MS scan followed by five MS/MS scans. Survey full-scan MS spectra were acquired in the orbitrap (m/z $350-2,000)$ with a resolution of 60,000 . Collision-induced dissociation (CID) fragmentation in the linear ion trap was performed for the five most intense ions at an automatic gain control target of 30,000 and a normalized collision energy of $38 \%$ at an activation of $q=0.25$ and an activation time of $30 \mathrm{~ms}$.

\section{Independent lipidomics analysis 2}

Homogenized membranes and protein crystals were extracted with $800 \mu \mathrm{L}$ of chloroform:methanol:water (1:1:0.1) in glass vials. After shaking the samples vigorously at 1500 rpm for $30 \mathrm{~min}$ at $4^{\circ} \mathrm{C}, 350 \mu \mathrm{L}$ of water was added to break the phases. Samples were centrifuged and the lower organic phase was transferred to new glass vials. The remaining membrane and/or protein pellets were re-extracted with 400 $\mu \mathrm{L}$ of chloroform. The combined extracts were dried using a SpeedVac (Genevac, UK). Samples were stored at $-80^{\circ} \mathrm{C}$ until mass spectrometric analysis.

Liquid chromatography-mass spectrometric (LCMS) analyses were carried out on an Exion UPLC coupled with a SciexQTRAP 6500 Plus mass spectrometer. Analysis of phospholipids and free mycolic acids were carried out using normal-phase LCMS as described previously $(62,63)$. Levels of menaquinone species were quantified using a reversephase LCMS method (64).

\section{Quinone reduction}

Menadiol was prepared as previously described, with minor modifications (65). To prepare reduced menadiol, $3.4 \mathrm{mg}$ 2-methyl-1,4-naphthoquinone (Sigma M5625) was dissolved in $1 \mathrm{~mL} \mathrm{~N}_{2}$-saturated anhydrous cyclohexane to yield a $20 \mathrm{mM}$ solution. The solution was mixed with $5 \mathrm{~mL} \mathrm{~N}_{2}$-saturated $1 \mathrm{M}$ sodium dithionite solution (in $\mathrm{H}_{2} \mathrm{O}$ ) and shaken vigorously. After phase separation, the organic phase containing the reduced menadiol was removed and transferred to a $15 \mathrm{~mL}$ centrifuge tube (all steps performed under a stream of $\mathrm{N}_{2}$ ). The cyclohexane was evaporated under an $\mathrm{N}_{2}$ stream, while the sample was kept at $40^{\circ} \mathrm{C}$ in a water bath. Subsequently, the reduced quinol was dissolved in $\mathrm{N}_{2}$-saturated, acidified ethanol (ethanol with $10 \mathrm{mM} \mathrm{HCl}$ ), aliquoted, flash frozen in liquid nitrogen, and stored at $-20^{\circ} \mathrm{C}$.

\section{Oxygen consumption assay}

Since the $b c c: a a_{3}$ preparations from $M$. smegmatis are active with menadiol (2-methyl-1,4-naphthoquinol) as substrate and electron donor, which has better solubility in water than menaquinol (33), the supercomplex was routinely characterized by its menadiol: $\mathrm{O}_{2}$ oxidoreductase activity. Rates of oxygen consumption were determined using a Clark-type oxygen electrode (Hansatech) in a magnetically stirred chamber at $25^{\circ} \mathrm{C}$ in $20 \mathrm{mM}$ MOPS pH 7.4, $100 \mathrm{mM} \mathrm{NaCl}, 0.005 \%$ LMNG, 
$5 \mathrm{mM}$ DTT, following a method previously described, with minor modifications (33). The rate of menadiol autoxidation was measured in parallel as a reference and subtracted from the supercomplex-dependent rate.

\section{Enzyme kinetics of supercomplex $\mathrm{CHI}_{2} \mathrm{CIV}_{2} \mathrm{SOD}_{2}$}

The Michaelis-Menten curves were obtained by measuring the initial supercomplex-dependent oxygen consumption rates as a function of menadiol concentration. The initial rate data were fitted to the Michealis-Menten equation using a non-linear fitting program (GraphPad Prism 6.0).

\section{In vitro inhibition of the supercomplex activity by Q203}

The supercomplexes were pre-incubated for $10 \mathrm{~min}$ with inhibitors, menadiol was added as electron donor to a final concentration of $200 \mu \mathrm{M}$ and the oxygen respiration was measured for $3 \mathrm{~min}$. Data were normalized relative to solvent (Ethanol) control for full activity and to a sample with 160 $\mu \mathrm{M}$ Antimycin A for complete inhibition.The supercomplexdependent initial rates were used to generate an inhibition curve and determine the $\mathrm{IC}_{50}$ value.

\section{Determination of SOD Activity in the supercomplex}

The superoxide dismutase (SOD) activity was measured using a SOD Assay Kit-WST (19160; Sigma-Aldrich) following the kit instruction. Since the absorption maximum of WST-1 formazan is $450 \mathrm{~nm}$ and it is proportional to the amount of superoxide anion, the SOD activity (inhibition rate \%) as an inhibition activity can be quantified by measuring the decrease in the color development at $450 \mathrm{~nm}$. The SOD activity of the complex purifications and a reference SOD sample with a known specifice activity in IU/mg (international unit per milligram) from bovine erythrocytes (Catalog number S5395; Sigma) were both determined in this assay. Based on the linear relationship between $1 /$ (inhibition rate) and $1 /($ enzyme activity), the $\mathrm{IC}_{50}$ (50\% inhibition activity) of the complex and bovine SOD can be determined. By comparing the $\mathrm{IC}_{50}$ of each, the specific SOD activity of the complex purification was determined and normalized into international unit per milligram. To be noted, the IU here is refer to the MF unit (McCord-Fridovich unit) which is determined by measuring the reduction of cytochrome $c$ by the xanthine oxidase/hypoxanthine system at $550 \mathrm{~nm}$ and is the international unit for SOD activity (EC 1.15.1.1) measurement (66). To avoid the potential interference from the cytochrome $c$ domains of QcrC subunite, McCord-Fridovich test was not choosen here.

\section{EPR spectroscopy}

Low temperature EPR spectra were recorded on a Bruker X-band (9.4 GHz) EMX plus 10/12 spectrometer equipped with an Oxford Instrument EPR 910 liquid Helium continuous-flow cryostat. A cylindrical resonator (ER4119hs TE011) was used for data collection. $100 \mu \mathrm{L}$ of supercomplex solution at $10 \mu \mathrm{M}$ was transferred to an EPR tube (Wilmad 707-SQ250M Quartz EPR sample tube) and rapidly frozen in liquid nitrogen before inserting into the resonator. For the reduced sample, sodium dithionite $(100 \mathrm{mM})$ was added and mixed with the supercomplex to give them spectrum of the reduced form. Specific parameters for EPR experiments were summarized here as: temperature $10 \mathrm{~K}$, microwave power $2.0 \mathrm{~mW}$, microwave frequency $9.39 \mathrm{GHz}$, modulation amplitude 0.9 $\mathrm{mT}$, modulation frequency $100 \mathrm{kHz}$, time constant $163.84 \mathrm{~ms}$, and scan rate $3.125 \mathrm{mT} / \mathrm{s}$. Multiple scans were accumulated to obtain a good $\mathrm{S} / \mathrm{N}$ ratio.

\section{Cryo-electron microscopy analysis}

Uranyl acetate $(1 \% \mathrm{w} / \mathrm{v})$ was used for negative staining. 5 $\mu \mathrm{L}$ of the supercomplex sample at concentration of $0.05 \mathrm{mg}$ $\mathrm{mL}^{-1}$ was applied to glow discharged copper grids supported by a thin layer of carbon film (Zhongjingkeyi Technology Co. Ltd) for one minute and stored at room temperature. Images were taken on an FEI Tecnai Spirit microscope operating at $120 \mathrm{kV}$. This data allowed initial model building.

Aliquots $(4 \mu \mathrm{L})$ of freshly purified supercomplex at a concentration of $0.5 \mathrm{mg} \mathrm{mL}^{-1}$ were applied to glow-discharged holey carbon grids (Quantifoil Au R1.2/1.3). The glow discharge was followed the standard recipts of $\mathrm{H}_{2}$ and $\mathrm{O}_{2}$ mixture in Gatan Solarus 950 for 1 min. Grids were blotted for $2.5 \mathrm{~s}$ and flash-frozen in liquid ethane cooled by liquid nitrogen using an FEI Mark IV Vitrobot operated at $8^{\circ} \mathrm{C}$ and $100 \%$ humidity. Images were taken using an FEI Titan Krios electron microscope operating at $300 \mathrm{kV}$ with a Gatan K2 Summit detector at a nominal magnification of $18,000 \times$. Images were recorded in super-resolution mode and binned to a pixel size of $1.35 \AA$. Automated single-particle data acquisition was performed with SerialEM (67). Defocus values varied from 1.3 to $2.7 \mu \mathrm{m}$. 32 frames per stack were collected with a total exposure time of $11.4 \mathrm{~s}$. The dose rate was set to $\sim 8 \mathrm{e}^{-} / \mathrm{pixel} / \mathrm{s}$ and the total dose was $\sim 50 \mathrm{e}^{-} / \AA^{2}$.

\section{Image processing}

We first generated a low-resolution reconstruction of the supercomplex from 53 micrographs of the negative-stained sample. Particle picking was performed with the EMAN2 (68) subroutine e2boxer.py in an interactive boxing mode, yielding 25,287 particles. Reference-free classification was performed with e2refine2d.py, generating 36 good classes from a total of 80 classes. The reconstruction model was generated by the good classes using e2initialmodel.py, which served as an initial model for the subsequent cryo-EM image processing. 
For Cryo-EM image processing, a total of 7,600 good micrographs were manually selected from 8,200 original micrographs. All processing steps were performed using Relion 1.4 or Relion 2.0 (69-72). A diagram of the procedures for data processing is presented in fig. $\mathrm{S} 3 \mathrm{C}$. At the whole-image level motion-corrected stacks were produced and binned 2 fold by MotionCorr (73) to produce motion-corrected stacks. Further motion correction and dose weighting was performed by MotionCorr2 (74) to average the output stacks. The contrast transfer function parameters of each image were estimated using Gctf (75). A total of 1,294,000 particles were automatically picked using Gautomatch (http://www.mrclmb.cam.ac.uk/kzhang/Gautomatch/). The particles were extracted using a $336^{2}$ pixel box and sorted by two rounds of reference-free $2 \mathrm{D}$ classification, resulting in $\sim 844,000$ particles selected from good 2D classes. These were subjected to $3 \mathrm{D}$ classification with the initial model that was low-pass filtered to $60 \AA$. The 3D classification was performed several times with different $\mathrm{K}$ values resulting in $\sim 400,000$ particles of good quality. The relative orientation of the QcrC subunit was variable, producing 3D classes with two different states. After 3D classification, the sets of particles within class-I and class-II were re-extracted and re-centered using a $292^{2}$ pixel box and subjected to the final refinement, respectively. The resolution of class-I particles was then corrected using the "high-resolution noise substitution" method (76) and estimated as $3.9 \AA$ according to the gold-standard FSC (Fourier shell correlation) 0.143 criterion. The pixel size was also calibrated to $1.30 \AA$ A $/$ pixel provided by the Center for Biological Imaging (CBI), Institute of Biophysics, Chinese Academy of Science. Then the resolution of the class-II particles was estimated to be $4.9 \AA$. Another $3 \mathrm{D}$ classification with class-I particles was performed by applying $\mathrm{C}_{2}$ symmetry, resulting in $\sim 202,000$ particles with good symmetry. The particles with $\mathrm{C}_{2}$ symmetry were further refined to yield a final reconstruction with the resolution of $3.5 \AA$ according to the gold-standard FSC 0.143 criterion. Prior to visualization, all density maps were corrected for the modulation transfer function (MTF) of the detector, and then sharpened by applying a negative B-factor (77) that was estimated using automated procedures. Local resolution variations were estimated using ResMap (78). The orientation distribution of the particles used in the final reconstruction was calculated using RELION 2.0.

\section{Model building and refinement}

All the subunits were initially built as poly-alanine chains, except for the residues that coordinate the prosthetic groups. Then residue assignment was performed, which was guided largely by secondary structure analysis carried out using PSIPRED (79) and Phyre2 (80).
It is noteworthy that the sequence of PRSAF1 in the model has an extra 16 amino acids before the $\mathrm{N}$ terminus of WP_003893930.1 but it is consistent with the revised complete ORF in the genome of $M$. smegmatis str. $\mathrm{MC}^{2} 51$ (genome locus tag AD56_RS0106885 added with the immediately preceding $64 \mathrm{bp}$ ). Model building was performed using Coot 0.8 (81) and further refined using the real space method in Phenix (82), except for the SOD catalytic domain (47Ala-236Gly) which was homology modeled (83) based on the structure of Sodc from M. tuberculosis (PDB Code: 1PZS) (36) and fitted into the map with the aid of the "Fit in Map" feature of Chimera (84). The completeness of the final resulting model was summarized in table S3.

For the phospholipids, the results of three independent mass spectrometry analyses confirmed that the majority variants of lipids in the purified sample are PE, PI and CL (fig. S1, L and N). No PC or other branching phospholipids was detected. Since most of the phospholipids are localized through the neighboring pipetides and are located in the transmembrane space which falls in the 3.2-3.5 $\AA$ high local resolution range, the CL moleculars were readily identified based on the four alkyl groups and the PI and PE molecules were distinguishable through the significent difference in their "head" group size. A CL molecular was also distinguishable from two PI or PE moleculars through the differnet distances between the two phosphatidic acid moieties. Finially, all the identified and assigned lipid backbones were modeled according to the EM potential map with necessary truncations and the results are summarized and illustrated against the context of the covering map in fig. S3I.

All the figures were created using PyMOL (85) or UCSF Chimera (84).

\section{REFERENCES AND NOTES}

1. P. Mitchell, Coupling of phosphorylation to electron and hydrogen transfer by a chemi-osmotic type of mechanism. Nature 191, 144-148 (1961). doi:10.1038/191144a0 Medline

2. A. M. Melo, M. Teixeira, Supramolecular organization of bacterial aerobic respiratory chains: From cells and back. Biochim. Biophys. Acta 1857, 190-197 (2016). doi:10.1016/j.bbabio.2015.11.001 Medline

3. E. A. Berry, B. L. Trumpower, Isolation of ubiquinol oxidase from Paracoccus denitrificans and resolution into cytochrome bcl and cytochrome c-aa3 complexes. J. Biol. Chem. 260, 2458-2467 (1985). Medline

4. J. A. Fee, M. G. Choc, K. L. Findling, R. Lorence, T. Yoshida, Properties of a coppercontaining cytochrome claa3 complex: A terminal oxidase of the extreme thermophile Thermus thermophilus HB8. Proc. NatI. Acad. Sci. U.S.A. 77, 147-151 (1980). doi:10.1073/pnas.77.1.147 Medline

5. J. Bengtsson, C. Rivolta, L. Hederstedt, D. Karamata, Bacillus subtilis contains two small c-type cytochromes with homologous heme domains but different types of membrane anchors. J. Biol. Chem. 274, 26179-26184 (1999). doi:10.1074/ibc.274.37.26179 Medline

6. M. Saraste, T. Metso, T. Nakari, T. Jalli, M. Lauraeus, J. Van der Oost, The Bacillus subtilis cytochrome-c oxidase. Variations on a conserved protein theme. Eur. J. Biochem. 195, 517-525 (1991). doi:10.1111/j.1432-1033.1991.tb15732.x Medline 
7. N. Sone, K. Nagata, H. Kojima, J. Tajima, Y. Kodera, T. Kanamaru, S. Noguchi, J. Sakamoto, A novel hydrophobic diheme c-type cytochrome. Purification from Corynebacterium glutamicum and analysis of the QcrCBA operon encoding three subunit proteins of a putative cytochrome reductase complex. Biochim. Biophys. Acta 1503, 279-290 (2001). doi:10.1016/S0005-2728(00)00205-X Medline

8. S. T. Cole, R. Brosch, J. Parkhill, T. Garnier, C. Churcher, D. Harris, S. V. Gordon, K. Eiglmeier, S. Gas, C. E. Barry 3rd, F. Tekaia, K. Badcock, D. Basham, D. Brown, T. Chillingworth, R. Connor, R. Davies, K. Devlin, T. Feltwell, S. Gentles, N. Hamlin, S. Holroyd, T. Hornsby, K. Jagels, A. Krogh, J. McLean, S. Moule, L. Murphy, K. Oliver, J. Osborne, M. A. Quail, M.-A. Rajandream, J. Rogers, S. Rutter, K. Seeger, J. Skelton, R. Squares, S. Squares, J. E. Sulston, K. Taylor, S. Whitehead, B. G. Barrell, Deciphering the biology of Mycobacterium tuberculosis from the complete genome sequence. Nature 393, 537-544 (1998). doi:10.1038/31159 Medline

9. J. A. Megehee, J. P. Hosler, M. D. Lundrigan, Evidence for a cytochrome bcc-aa3 interaction in the respiratory chain of Mycobacterium smegmatis. Microbiology 152, 823-829 (2006). doi:10.1099/mic.0.28723-0 Medline

10. W. C. Kao, T. Kleinschroth, W. Nitschke, F. Baymann, Y. Neehaul, P. Hellwig, S. Richers, J. Vonck, M. Bott, C. Hunte, The obligate respiratory supercomplex from Actinobacteria. Biochim. Biophys. Acta 1857, 1705-1714 (2016). doi:10.1016/i.bbabio.2016.07.009 Medline

11. C. Sun, S. Benlekbir, P. Venkatakrishnan, Y. Wang, S. Hong, J. Hosler, E. Tajkhorshid, J. L. Rubinstein, R. B. Gennis, Structure of the alternative complex III in a supercomplex with cytochrome oxidase. Nature 557, 123-126 (2018). doi:10.1038/s41586-018-0061-y Medline

12. A. Puustinen, M. Finel, T. Haltia, R. B. Gennis, M. Wikström, Properties of the two terminal oxidases of Escherichia coli. Biochemistry 30, 3936-3942 (1991). doi:10.1021/bi00230a019 Medline

13. A. L. Moore, M. S. Albury, Further insights into the structure of the alternative oxidase: From plants to parasites. Biochem. Soc. Trans. 36, 1022-1026 (2008). doi:10.1042/BST0361022 Medline

14. R. Baradaran, J. M. Berrisford, G. S. Minhas, L. A. Sazanov, Crystal structure of the entire respiratory complex I. Nature 494, 443-448 (2013). doi:10.1038/nature11871 Medline

15. K. R. Vinothkumar, J. Zhu, J. Hirst, Architecture of mammalian respiratory complex I. Nature 515, 80-84 (2014). doi:10.1038/nature13686 Medline

16. J. Zhu, K. R. Vinothkumar, J. Hirst, Structure of mammalian respiratory complex I. Nature 536, 354-358 (2016). doi:10.1038/nature19095 Medline

17. V. Yankovskaya, R. Horsefield, S. Törnroth, C. Luna-Chavez, H. Miyoshi, C. Léger, B. Byrne, G. Cecchini, S. Iwata, Architecture of succinate dehydrogenase and reactive oxygen species generation. Science 299, 700-704 (2003). doi:10.1126/science.1079605 Medline

18. F. Sun, X. Huo, Y. Zhai, A. Wang, J. Xu, D. Su, M. Bartlam, Z. Rao, Crystal structure of mitochondrial respiratory membrane protein complex II. Cell 121, 1043-1057 (2005). doi:10.1016/i.cell.2005.05.025 Medline

19. D. Xia, C.-A. Yu, H. Kim, J.-Z. Xia, A. M. Kachurin, L. Zhang, L. Yu, J. Deisenhofer, Crystal structure of the cytochrome bcl complex from bovine heart mitochondria. Science 277, 60-66 (1997). doi:10.1126/science.277.5322.60 Medline

20. S. Iwata, J. W. Lee, K. Okada, J. K. Lee, M. Iwata, B. Rasmussen, T. A. Link, S. Ramaswamy, B. K. Jap, Complete structure of the 11-subunit bovine mitochondrial cytochrome bcl complex. Science 281, 64-71 (1998). doi:10.1126/science.281.5373.64 Medline

21. Z. Zhang, L. Huang, V. M. Shulmeister, Y.-I. Chi, K. K. Kim, L.-W. Hung, A. R. Crofts, E. A. Berry, S.-H. Kim, Electron transfer by domain movement in cytochrome bc1. Nature 392, 677-684 (1998). doi:10.1038/33612 Medline

22. S. Iwata, C. Ostermeier, B. Ludwig, H. Michel, Structure at 2.8 A resolution of cytochrome c oxidase from Paracoccus denitrificans. Nature 376, 660-669 (1995). doi:10.1038/376660a0 Medline

23. T. Tsukihara, H. Aoyama, E. Yamashita, T. Tomizaki, H. Yamaguchi, K. ShinzawaItoh, R. Nakashima, R. Yaono, S. Yoshikawa, The whole structure of the 13-subunit oxidized cytochrome c oxidase at 2.8 A. Science 272, 1136-1144 (1996). doi:10.1126/science. 272.5265 .1136 Medline

24. S. Yoshikawa, K. Shinzawa-Itoh, R. Nakashima, R. Yaono, E. Yamashita, N. Inoue, M. Yao, M. J. Fei, C. P. Libeu, T. Mizushima, H. Yamaguchi, T. Tomizaki, T. Tsukihara, Redox-coupled crystal structural changes in bovine heart cytochrome c oxidase. Science 280, 1723-1729 (1998). doi:10.1126/science.280.5370.1723 Medline

25. J. A. Letts, K. Fiedorczuk, L. A. Sazanov, The architecture of respiratory supercomplexes. Nature 537, 644-648 (2016). doi:10.1038/nature19774 Medline

26. E. Mileykovskaya, P. A. Penczek, J. Fang, V. K. P. S. Mallampalli, G. C. Sparagna, W. Dowhan, Arrangement of the respiratory chain complexes in Saccharomyces cerevisiae supercomplex III2IV2 revealed by single particle cryo-electron microscopy. J. Biol. Chem. 287, 23095-23103 (2012) doi:10.1074/ibc.M112.367888 Medline

27. J. A. Letts, L. A. Sazanov, Clarifying the supercomplex: The higher-order organization of the mitochondrial electron transport chain. Nat. Struct. Mol. Biol. 24, 800-808 (2017). doi:10.1038/nsmb.3460 Medline

28. D. Milenkovic, J. N. Blaza, N. G. Larsson, J. Hirst, The enigma of the respiratory chain supercomplex. Cell Metab. 25, 765-776 (2017) doi:10.1016/i.cmet.2017.03.009 Medline

29. J. D. Chavez, C. F. Lee, A. Caudal, A. Keller, R. Tian, J. E. Bruce, Chemical crosslinking mass spectrometry analysis of protein conformations and supercomplexes in heart tissue. Cell Syst. 6, 136-141.e5 (2018). do::10.1016/i.cels.2017.10.017 Medline

30. K. M. Davies, T. B. Blum, W. Kühlbrandt, Conserved in situ arrangement of complex I and $\mathrm{II}_{2}$ in mitochondrial respiratory chain supercomplexes of mammals, yeast, and plants. Proc. Natl. Acad. Sci. U.S.A. 115, 3024-3029 (2018). doi:10.1073/pnas. 1720702115 Medline

31. A. Niebisch, M. Bott, Molecular analysis of the cytochrome bc1-aa3 branch of the Corynebacterium glutamicum respiratory chain containing an unusual diheme cytochrome c1. Arch. Microbiol. 175, 282-294 (2001). doi:10.1007/s002030100262 Medline

32. M. S. Kim, J. Jang, N. B. Ab Rahman, K. Pethe, E. A. Berry, L.-S. Huang, Isolation and characterization of a hybrid respiratory supercomplex consisting of Mycobacterium tuberculosis cytochrome bcc and Mycobacterium smegmatis cytochrome aa3. J. Biol. Chem. 290, 14350-14360 (2015). doi:10.1074/ibc.M114.624312 Medline

33. E. Lemma, H. Schägger, A. Kröger, The menaquinol oxidase of Bacillus subtilis W23. Arch. Microbiol. 159, 574-578 (1993). doi:10.1007/BF00249037 Medline

34. M. M. Babu, M. L. Priya, A. T. Selvan, M. Madera, J. Gough, L. Aravind, K. Sankaran, A database of bacterial lipoproteins (DOLOP) with functional assignments to predicted lipoproteins. J. Bacteriol. 188, 2761-2773 (2006). doi:10.1128/JB.188.8.2761-2773.2006 Medline

35. M. D'orazio, S. Folcarelli, F. Mariani, V. Colizzi, G. Rotilio, A. Battistoni, Lipid modification of the $\mathrm{Cu}, \mathrm{Zn}$ superoxide dismutase from Mycobacterium tuberculosis. Biochem. J. 359, 17-22 (2001). doi:10.1042/bj3590017 Medline

36. L. Spagnolo, I. Törö, M. D'Orazio, P. O'Neill, J. Z. Pedersen, O. Carugo, G. Rotilio, A. Battistoni, K. Djinović-Carugo, Unique features of the sodC-encoded superoxide dismutase from Mycobacterium tuberculosis, a fully functional copper-containing enzyme lacking zinc in the active site. J. Biol. Chem. 279, 33447-33455 (2004). doi:10.1074/jbc.M404699200 Medline

37. F. L. Sousa, R. J. Alves, M. A. Ribeiro, J. B. Pereira-Leal, M. Teixeira, M. M. Pereira, The superfamily of heme-copper oxygen reductases: Types and evolutionary considerations. Biochim. Biophys. Acta 1817, 629-637 (2012). doi:10.1016/i.bbabio.2011.09.020 Medline

38. M. Svensson-Ek, J. Abramson, G. Larsson, S. Törnroth, P. Brzezinski, S. Iwata, The $X$-ray crystal structures of wild-type and EQ(I-286) mutant cytochrome c oxidases from Rhodobacter sphaeroides. J. Mol. Biol. 321, 329-339 (2002). doi:10.1016/S0022-2836(02)00619-8 Medline

39. A. Magalon, R. Arias-Cartin, A. Walburger, Supramolecular organization in prokaryotic respiratory systems. Adv. Microb. Physiol. 61, 217-266 (2012). doi:10.1016/B978-0-12-394423-8.00006-8 Medline

40. D. Birth, W. C. Kao, C. Hunte, Structural analysis of atovaquone-inhibited cytochrome bcl complex reveals the molecular basis of antimalarial drug action. Nat. Commun. 5, 4029 (2014). doi:10.1038/ncomms5029 Medline

41. K. Pethe, P. Bifani, J. Jang, S. Kang, S. Park, S. Ahn, J. Jiricek, J. Jung, H. K. Jeon, J. Cechetto, T. Christophe, H. Lee, M. Kempf, M. Jackson, A. J. Lenaerts, H. Pham, V. Jones, M. J. Seo, Y. M. Kim, M. Seo, J. J. Seo, D. Park, Y. Ko, I. Choi, R. Kim, S. Y. Kim, S. Lim, S.-A. Yim, J. Nam, H. Kang, H. Kwon, C.-T. Oh, Y. Cho, Y. Jang, J. Kim, 
A. Chua, B. H. Tan, M. B. Nanjundappa, S. P. S. Rao, W. S. Barnes, R. Wintjens, J. R. Walker, S. Alonso, S. Lee, J. Kim, S. Oh, T. Oh, U. Nehrbass, S.-J. Han, Z. No, J. Lee, P. Brodin, S.-N. Cho, K. Nam, J. Kim, Discovery of Q203, a potent clinical candidate for the treatment of tuberculosis. Nat. Med. 19, 1157-1160 (2013). doi:10.1038/nm.3262 Medline

42. P. Lu, A. H. Asseri, M. Kremer, J. Maaskant, R. Ummels, H. Lill, D. Bald, The antimycobacterial activity of the cytochrome bcc inhibitor Q203 can be enhanced by small-molecule inhibition of cytochrome bd. Sci. Rep. 8, 2625 (2018). doi:10.1038/s41598-018-20989-8 Medline

43. M. Swierczek, E. Cieluch, M. Sarewicz, A. Borek, C. C. Moser, P. L. Dutton, A. Osyczka, An electronic bus bar lies in the core of cytochrome bcl. Science 329, 451-454 (2010). doi:10.1126/science. 1190899 Medline

44. H. Witt, F. Malatesta, F. Nicoletti, M. Brunori, B. Ludwig, Tryptophan 121 of subunit $\|$ is the electron entry site to cytochrome-c oxidase in Paracoccus denitrificans. Involvement of a hydrophobic patch in the docking reaction. J. Biol. Chem. 273, 5132-5136 (1998). doi:10.1074/jbc.273.9.5132 Medline

45. S. Shimada, K. Shinzawa-Itoh, J. Baba, S. Aoe, A. Shimada, E. Yamashita, J. Kang, M. Tateno, S. Yoshikawa, T. Tsukihara, Complex structure of cytochrome ccytochrome coxidase reveals a novel protein-protein interaction mode. EMBO J. 36, 291-300 (2017). doi:10.15252/embj.201695021 Medline

46. T. Soulimane, G. Buse, G. P. Bourenkov, H. D. Bartunik, R. Huber, M. E. Than, Structure and mechanism of the aberrant ba ((3))-cytochrome $\mathrm{c}$ oxidase from thermus thermophilus. EMBO J. 19, 1766-1776 (2000). doi:10.1093/emboj/19.8.1766 Medline

47. C. Ostermeier, A. Harrenga, U. Ermler, H. Michel, Structure at 2.7 A resolution of the Paracoccus denitrificans two-subunit cytochrome $c$ oxidase complexed with an antibody FV fragment. Proc. Natl. Acad. Sci. U.S.A. 94, 10547-10553 (1997). doi:10.1073/pnas.94.20.10547 Medline

48. F. Millett, B. Durham, Chapter 5 Use of ruthenium photooxidation techniques to study electron transfer in the cytochrome bcl complex. Methods Enzymol. 456, 95-109 (2009). doi:10.1016/S0076-6879(08)04405-4 Medline

49. C. C. Moser, J. M. Keske, K. Warncke, R. S. Farid, P. L. Dutton, Nature of biological electron transfer. Nature 355, 796-802 (1992). doi:10.1038/355796a0 Medline

50. S. Bhattacharya, Reactive Oxygen Species and Cellular Defense System. (2015), pp. 17-29.

51. J. F. Turrens, A. Boveris, Generation of superoxide anion by the NADH dehydrogenase of bovine heart mitochondria. Biochem. J. 191, 421-427 (1980). doi:10.1042/bj1910421 Medline

52. X. Zhao, K. Drlica, Reactive oxygen species and the bacterial response to lethal stress. Curr. Opin. Microbiol. 21, 1-6 (2014). doi:10.1016/j.mib.2014.06.008 Medline

53. G. Petrosillo, F. M. Ruggiero, N. Di Venosa, G. Paradies, Decreased complex III activity in mitochondria isolated from rat heart subjected to ischemia and reperfusion: Role of reactive oxygen species and cardiolipin. FASEB J. 17, 714-716 (2003). doi:10.1096/fi.02-0729fje Medline

54. G. Paradies, G. Petrosillo, M. Pistolese, F. M. Ruggiero, The effect of reactive oxygen species generated from the mitochondrial electron transport chain on the cytochrome $\mathrm{C}$ oxidase activity and on the cardiolipin content in bovine heart submitochondrial particles. FEBS Lett. 466, 323-326 (2000). doi:10.1016/S0014-5793(00)01082-6 Medline

55. G. Paradies, G. Petrosillo, V. Paradies, F. M. Ruggiero, Oxidative stress, mitochondrial bioenergetics, and cardiolipin in aging. Free Radic. Biol. Med. 48, 1286-1295 (2010). doi:10.1016/j.freeradbiomed.2010.02.020 Medline

56. D. L. Piddington, F. C. Fang, T. Laessig, A. M. Cooper, I. M. Orme, N. A. Buchmeier, $\mathrm{Cu}, \mathrm{Zn}$ superoxide dismutase of Mycobacterium tuberculosis contributes to survival in activated macrophages that are generating an oxidative burst. Infect. Immun. 69, 4980-4987 (2001). doi:10.1128/IAl.69.8.4980-4987.2001 Medline

57. W. Suthammarak, B. H. Somerlot, E. Opheim, M. Sedensky, P. G. Morgan, Novel interactions between mitochondrial superoxide dismutases and the electron transport chain. Aging Cell 12, 1132-1140 (2013). doi:10.1111/acel.12144 Medline

58. X. Li, F. Liu, Y. Hu, K. Mi, Draft genome sequence of $m c^{2} 51$, a highly hydrogen peroxide-resistant Mycobacterium smegmatis mutant strain. Genome Announc. 2, e00092-14 (2014). doi:10.1128/genomeA.00092-14 Medline

59. I. Wittig, H. P. Braun, H. Schägger, Blue native PAGE. Nat. Protoc. 1, 418-428 (2006). doi:10.1038/nprot.2006.62 Medline
60. I. Wittig, H. Schägger, Features and applications of blue-native and clear-native electrophoresis. Proteomics 8, 3974-3990

(2008). doi:10.1002/pmic.200800017Medline

61. L. Y. J. García Montes de Oca, A. Chagolla-López, L. González de la Vara, T. Cabellos-Avelar, C. Gómez-Lojero, E. B. Gutiérrez Cirlos, The composition of the Bacillus subtilis aerobic respiratory chain supercomplexes. J. Bioenerg. Biomembr. 44, 473-486 (2012). doi:10.1007/s10863-012-9454-z Medline

62. S. M. Lam, L. Tong, X. Duan, A. Petznick, M. R. Wenk, G. Shui, Extensive characterization of human tear fluid collected using different techniques unravels the presence of novel lipid amphiphiles. J. Lipid Res. 55, 289-298 (2014). doi:10.1194/jle.M044826 Medline

63. G. Shui, A. K. Bendt, I. A. Jappar, H. M. Lim, M. Laneelle, M. Hervé, L. E. Via, G. H. Chua, M. W. Bratschi, S. Z. Zainul Rahim, A. L. T. Michelle, S.-H. Hwang, J.-S. Lee, S.-Y. Eum, H.-K. Kwak, M. Daffé, V. Dartois, G. Michel, C. E. Barry 3rd, M. R. Wenk, Mycolic acids as diagnostic markers for tuberculosis case detection in humans and drug efficacy in mice. EMBO Mol. Med. 4, 27-37 (2012). doi:10.1002/emmm.201100185 Medline

64. L. Lim, V. Jackson-Lewis, L. C. Wong, G. H. Shui, A. X. H. Goh, S. Kesavapany, A. M. Jenner, M. Fivaz, S. Przedborski, M. R. Wenk, Lanosterol induces mitochondrial uncoupling and protects dopaminergic neurons from cell death in a model for Parkinson's disease. Cell Death Differ. 19, 416-427 (2012). doi:10.1038/cdd.2011.105 Medline

65. S. Graf, O. Fedotovskaya, W.-C. Kao, C. Hunte, P. Ädelroth, M. Bott, C. von Ballmoos, P. Brzezinski, Rapid electron transfer within the III-IV supercomplex in Corynebacterium glutamicum. Sci. Rep. 6, 34098 (2016). doi:10.1038/srep34098 Medline

66. J. M. McCord, I. Fridovich, Superoxide dismutase. An enzymic function for erythrocuprein (hemocuprein). J. Biol. Chem. 244, 6049-6055 (1969). Medline

67. D. N. Mastronarde, Automated electron microscope tomography using robust prediction of specimen movements. J. Struct. Biol. 152, 36-51 (2005). doi:10.1016/i.jsb.2005.07.007 Medline

68. G. Tang, L. Peng, P. R. Baldwin, D. S. Mann, W. Jiang, I. Rees, S. J. Ludtke, EMAN2: An extensible image processing suite for electron microscopy. J. Struct. Biol. 157 38-46 (2007). doi:10.1016/j.jsb.2006.05.009 Medline

69. S. H. Scheres, Semi-automated selection of cryo-EM particles in RELION-1.3. J. Struct. Biol. 189, 114-122 (2015). doi:10.1016/j.jsb.2014.11.010 Medline

70. S. H. Scheres, A Bayesian view on cryo-EM structure determination. J. Mol. Biol. 415, 406-418 (2012). doi:10.1016/j.jmb.2011.11.010 Medline

71. S. H. Scheres, RELION: Implementation of a Bayesian approach to cryo-EM structure determination. J. Struct. Biol. 180, 519-530 (2012). doi:10.1016/j.jsb.2012.09.006 Medline

72. D. Kimanius, B. O. Forsberg, S. H. Scheres, E. Lindahl, Accelerated cryo-EM structure determination with parallelisation using GPUs in RELION-2. eLife 5, e18722 (2016). doi:10.7554/eLife.18722 Medline

73. X. Li, P. Mooney, S. Zheng, C. R. Booth, M. B. Braunfeld, S. Gubbens, D. A. Agard, $Y$. Cheng, Electron counting and beam-induced motion correction enable nearatomic-resolution single-particle cryo-EM. Nat. Methods 10, 584-590 (2013). do: $10.1038 /$ nmeth.2472 Medline

74. S. Q. Zheng, E. Palovcak, J.-P. Armache, K. A. Verba, Y. Cheng, D. A. Agard, MotionCor2: Anisotropic correction of beam-induced motion for improved cryoelectron microscopy. Nat. Methods 14, 331-332 (2017). doi:10.1038/nmeth.4193 Medline

75. K. Zhang, Gctf: Real-time CTF determination and correction. J. Struct. Biol. 193, 112 (2016). doi:10.1016/j.jsb.2015.11.003 Medline

76. S. Chen, G. McMullan, A. R. Faruqi, G. N. Murshudov, J. M. Short, S. H. W. Scheres, R. Henderson, High-resolution noise substitution to measure overfitting and validate resolution in 3D structure determination by single particle electron cryomicroscopy. Ultramicroscopy 135, 24-35 doi:10.1016/j.ultramic.2013.06.004 Medline

77. P. B. Rosenthal, R. Henderson, Optimal determination of particle orientation, absolute hand, and contrast loss in single-particle electron cryomicroscopy. J. Mol. Biol. 333, 721-745 (2003). doi:10.1016/j.jmb.2003.07.013 Medline

78. A. Kucukelbir, F. J. Sigworth, H. D. Tagare, Quantifying the local resolution of cryoEM density maps. Nat. Methods 11, 63-65 (2014). doi:10.1038/nmeth.2727 Medline 
79. L. J. McGuffin, K. Bryson, D. T. Jones, The PSIPRED protein structure prediction server. Bioinformatics 16, 404-405 (2000). doi:10.1093/bioinformatics/16.4.404 Medline

80. L. A. Kelley, S. Mezulis, C. M. Yates, M. N. Wass, M. J. Sternberg, The Phyre2 web portal for protein modeling, prediction and analysis. Nat. Protoc. 10, 845-858 (2015). doi:10.1038/nprot.2015.053 Medline

81. P. Emsley, B. Lohkamp, W. G. Scott, K. Cowtan, Features and development of Coot. Acta Crystallogr. D Biol. Crystallogr. 66, 486-501 (2010). doi:10.1107/S0907444910007493 Medline

82. P. D. Adams, P. V. Afonine, G. Bunkóczi, V. B. Chen, I. W. Davis, N. Echols, J. J. Headd, L.-W. Hung, G. J. Kapral, R. W. Grosse-Kunstleve, A. J. McCoy, N. W. Moriarty, R. Oeffner, R. J. Read, D. C. Richardson, J. S. Richardson, T. C. Terwilliger, P. H. Zwart, PHENIX: A comprehensive Python-based system for macromolecular structure solution. Acta Crystallogr. D Biol. Crystallogr. 66, 213221 (2010). doi:10.1107/S0907444909052925 Medline

83. J. Yang, R. Yan, A. Roy, D. Xu, J. Poisson, Y. Zhang, The I-TASSER Suite: Protein structure and function prediction. Nat. Methods 12, 7-8 (2015). doi:10.1038/nmeth.3213 Medline

84. E. F. Pettersen, T. D. Goddard, C. C. Huang, G. S. Couch, D. M. Greenblatt, E. C. Meng, T. E. Ferrin, UCSF Chimera-a visualization system for exploratory research and analysis. J. Comput. Chem. 25, 1605-1612 (2004). doi:10.1002/jec.20084 Medline

85. W. L. Delano, The PyMOL Molecular Graphics System (DeLano Scientific, 2002).

86. N. Sone, Y. Yanagita, A cytochrome aa3-type terminal oxidase of a thermophilic bacterium. Purification, properties and proton pumping. Bioenergetics $682,216-$ 226 (1982). doi:10.1016/0005-2728(82)90101-3

87. B. Chance, in Methods in Enzymology. (Academic Press, 1957), vol. 4, pp. 273329.

88. M. J. Sartain, J. T. Belisle, N-Terminal clustering of the 0-glycosylation sites in the Mycobacterium tuberculosis lipoprotein SodC. Glycobiology 19, 38-51 (2009). doi:10.1093/glycob/cwn102 Medline

89. L. Esser, F. Zhou, Y. Zhou, Y. Xiao, W. K. Tang, C.-A. Yu, Z. Qin, D. Xia, Hydrogen bonding to the substrate Is not required for rieske iron-sulfur protein docking to the quinol oxidation site of complex III. J. Biol. Chem. 291, 25019-25031 (2016). doi:10.1074/ibc.M116.744391 Medline

90. J. Koepke, E. Olkhova, H. Angerer, H. Müller, G. Peng, H. Michel, High resolution crystal structure of Paracoccus denitrificans cytochrome c oxidase: New insights into the active site and the proton transfer pathways. Biochim. Biophys. Acta1787, 635-645 (2009). doi:10.1016/j.bbabio.2009.04.003 Medline

91. L. Holm, L. M. Laakso, Dali server update. Nucleic Acids Res. 44, W351-W355 (2016). doi:10.1093/nar/gkw357Medline

92. J. Felsenstein, PHYLIP - Phylogeny Inference Package (Version 3.2). Cladistics 5 , 164-166 (1989).

93. F. D. Ciccarelli, T. Doerks, C. von Mering, C. J. Creevey, B. Snel, P. Bork, Toward automatic reconstruction of a highly resolved tree of life. Science 311, 1283-1287 (2006). doi:10.1126/science.1123061 Medline

94. K. L. Smollett, A. S. Fivian-Hughes, J. E. Smith, A. Chang, T. Rao, E. O. Davis, Experimental determination of translational start sites resolves uncertainties in genomic open reading frame predictions - application to Mycobacterium tuberculosis. Microbiology 155, 186-197 (2009). doi:10.1099/mic.0.022889-0 Medline

\section{ACKNOWLEDGMENTS}

We would like to thank Jianchao Zhang, Yini Zhang and Dr. Lei Wang from Prof. ChihChen Wang's Research Group (National Laboratory of Biomacromolecules, Institute of Biophysics, CAS) for their technical support on Clark-type oxygen electrode and oxygen consumption assay and Prof. Kaixia Mi (CAS Key Laboratory of Pathogenic Microbiology and Immunology, Institute of Microbiology, CAS) for sharing the strain M. smegmatis $\mathrm{mc}^{2} 51$. We are also grateful to Boling Zhu, Xiaojun Huang and Gang Ji from Center for Biological Imaging (CBI), Institute of Biophysics, CAS and staff members from National Center for Protein Science Shanghai (NCPSS) for their technical support on cryo-EM and Dr. Chao Peng from the Mass Spectrometry System of NCPSS for his technical support. We also thank Tongxin Niu from the HPC-Service Station in $\mathrm{CB}$ and Xiaodong Jian and Xiangfei Meng from TianHe-1(A) at National
Supercomputer Center in Tianjin for computational support. Funding: This work was supported by grants from the National Key Research and Development Program of China (Grant No. 2017YFC0840300), the Strategic Priority Research Program of the Chinese Academy of Sciences (Grant No. XDB08020200), the State Key Development Program for Basic Research of the Ministry of Science and Technology of China (973 Project Grant Nos. 2014CB542800, 2014 CBA02003 to ZR and 2014CB910700 to FS), and the National Natural Science Foundation of China (Grant Nos. 813300237 and 81520108019). Author contributions: Z.R. conceived, initiated and coordinated the project. H.G., Ju.L and S.W. purified the M. smegmatis SC III-IV supercomplex; H.G. Y.T. and Ju.L. characterized the spectroscopic features of the samples; Ji.L., H.Y., C.R., S.L. and G.S. preformed mass spectrometry analysis and identified the contents of the complex; Y.T. and Q.W. set up the biochemical assays and measured the menadiol: $\mathrm{O}_{2}$ oxidoreductase activity of the complex; H.G. measured the superoxide dismutase activity of the complex; L.Y. and C.T. performed the EPR experiments and data analysis; A.X. and Q.W. collected and processed cryo-EM data; Q.W. reconstructed the $3.5 \AA$ resolution map and supervised cryo-EM structure determination; O.W., A.X., R.G. and W.J. built and refined the structure model; Ju.L., Q.W., F.S., H.G, A.X., X.Y., Y.S, X.L., M.J., C.T., C.Y., B.J., Z.L., L.G., L.W. and Z.R. analyzed the structure and discussed the results and the manuscript was written by F.S., Q.W., Ju.L., W.J., A.X., H.G., R.G., Z.L., L.G., L.W. and Z.R. Competing interests: The authors declare no competing interests. L.W. is a founder and consultant of Oxford Biotrans Ltd., UK. C.R. is a founding director and consultant of OMass Technologies Ltd., UK. Data and materials availability: All data are available in the manuscript or the supplementary material. The accesion number for the 3D cryo-EM density map reported in this paper is EMD-9610. The PDB accession number for the coordinates of the CIIICIV complex is 6ADQ.

\section{SUPPLEMENTARY MATERIALS}

www.sciencemag.org/cgi/content/full/science.aat8923/DC1

Figs. S1 to S8

Tables $\mathrm{S} 1$ to $\mathrm{S} 4$

References (86-94)

Movie S1

16 April 2018; accepted 10 October 2018

Published online 25 October 2018

$10.1126 /$ science.aat8923 
A

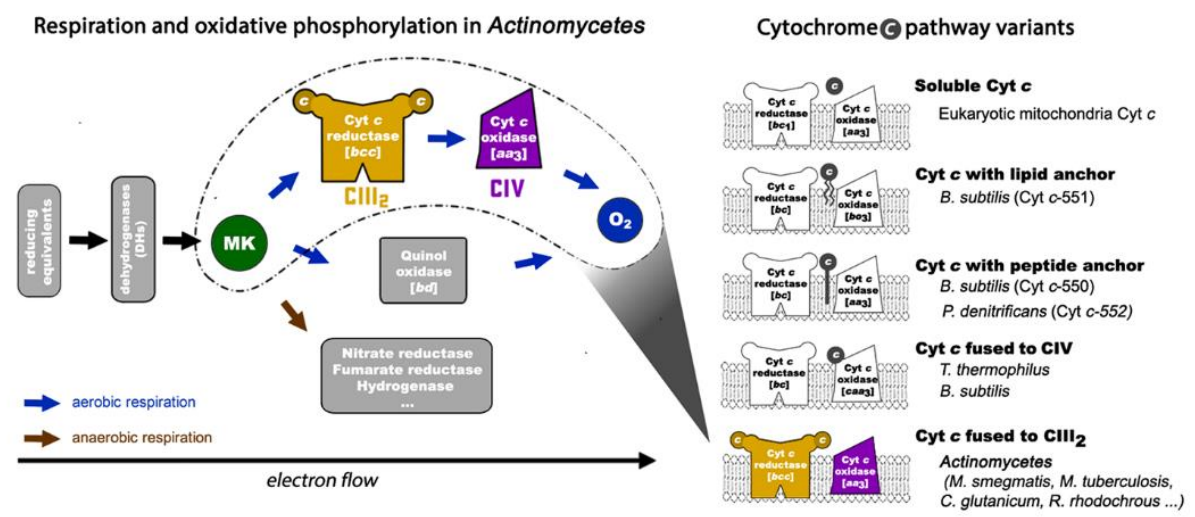

B

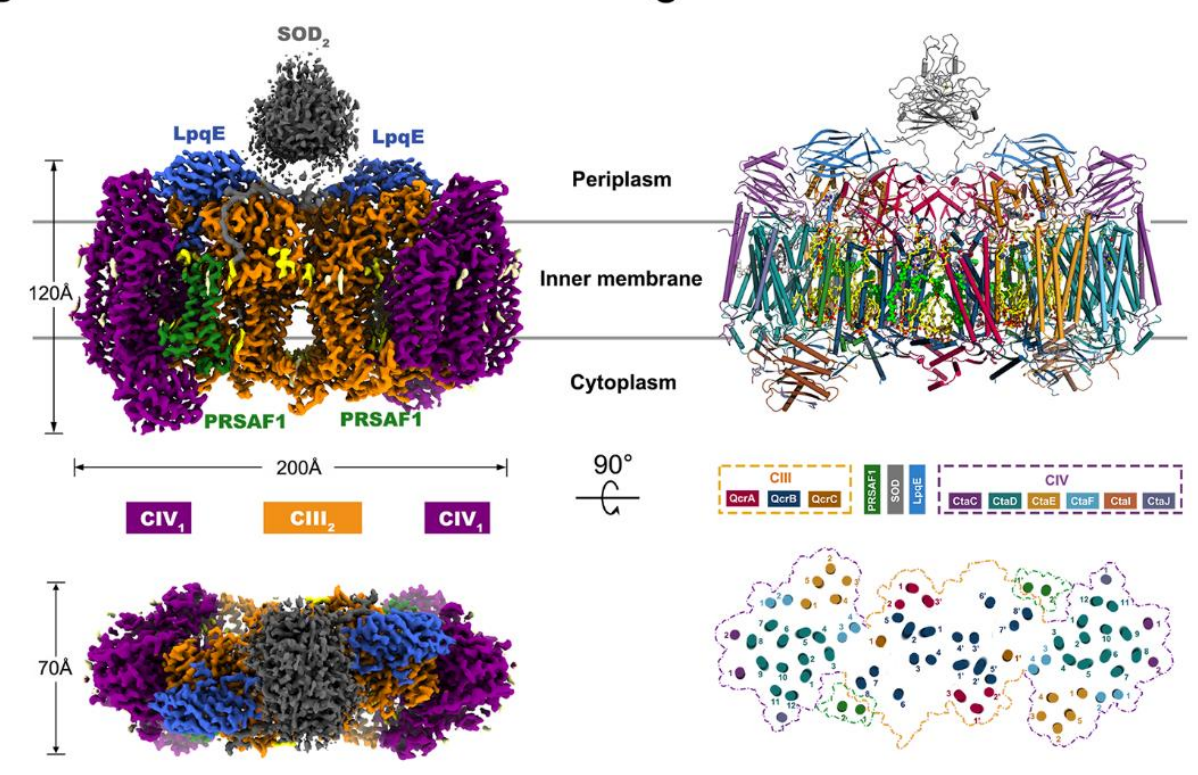

Fig. 1. Respiration in Actinomycetes and overall architecture of the Mycobacterial respiratory machine $\mathrm{ClII}_{2} \mathrm{CIV}_{2} \mathrm{SOD}_{2}$. (A) The respiratory electron transfer chain in Actinomycetes (left) and the five major prokaryotic cytochrome $c$ pathway variants with the organization schemes in representative organisms (right). The $M$. smegmatis cytochrome $c$ pathway has its cytochrome $c$ fused with complex III, forming a bcc-type complex III that interacts with the $a a_{3}$-type complex IV to assemble into a CIII-CIV supercomplex.

$\mathrm{MK}=$ menaquinone/menaquinol.

(B) Overall architecture of the bcc$\mathrm{aa}_{3}$ type respiratory CIII-CIV supercomplex from $M$. smegmatis. The cryo-EM map of the supercomplex shows a linear twofold dimerized form of $\mathrm{CIV}_{1}-\mathrm{CII}_{2}-$ $\mathrm{CIV}_{1}$ with dimensions $200 \times 70 \times$ $120 \AA$. CIII is colored in orange, CIV in magenta and the association factors, PRSAF1 in green, LpqE in blue and SOD in grey. (C) Cartoon representation of the side view of the supercomplex (top) and a cross sectional view (bottom). The menaquinone/menaquinol ( $\mathrm{MK}$ ) is presented as bright green colored solid spheres and the phospholipids as yellow sticks. In the cross-sectional view (bottom), the boundaries of CIII, CIV and the association factor PRSAF1 are depicted with dashed lines in color (orange for CIII, magenta for CIV and green for PRSAF1). 

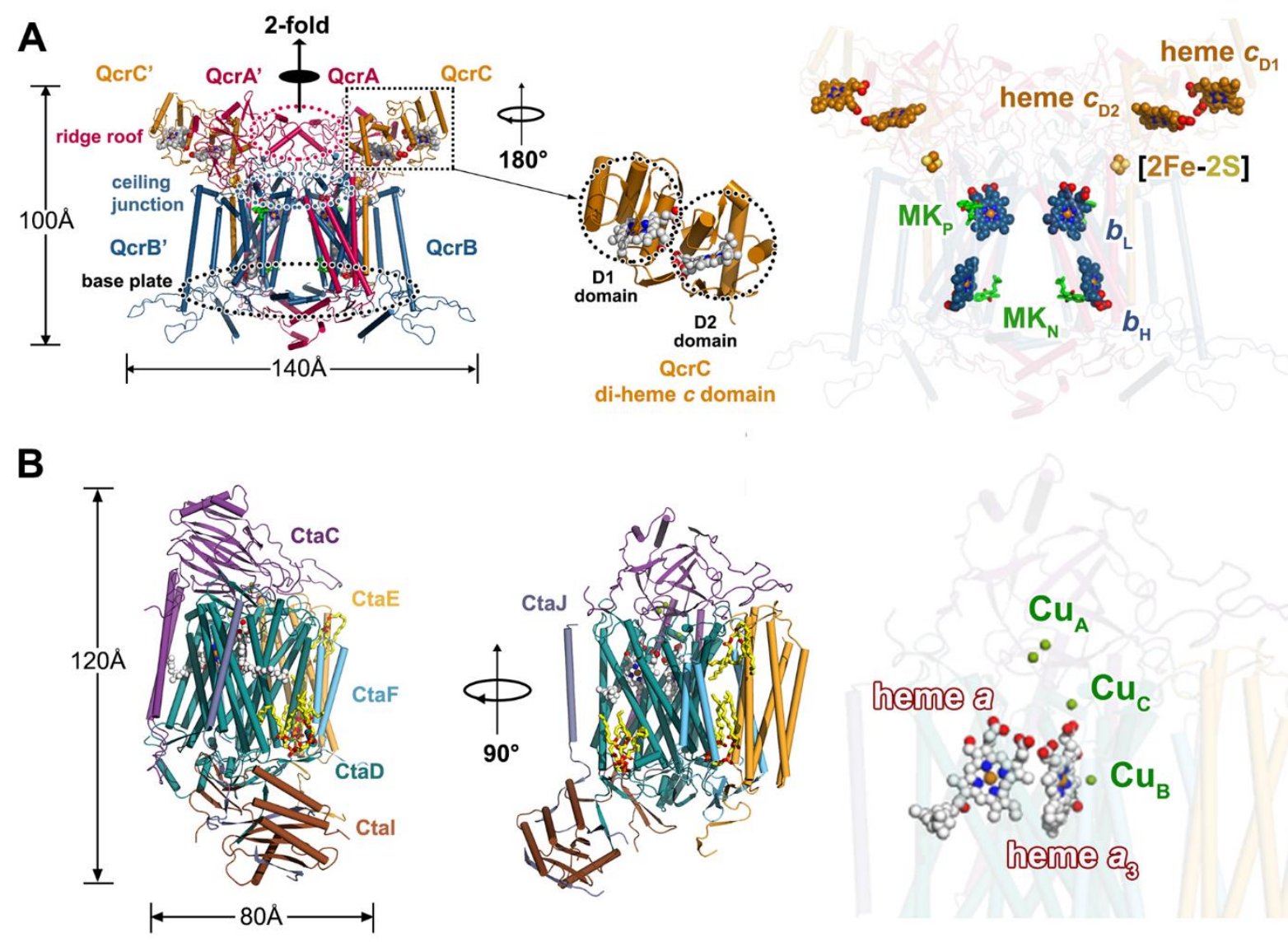

Fig. 2. Structure of $\mathrm{CIII}_{2}$ and CIV from M. smegmatis. (A) Overall structure of the CIII dimer (left) and the spatial location (right) of prosthetic groups. QcrA, QcrB and QcrC are colored pink, blue and gold, respectively. The two-fold symmetry of the dimer is depicted by the black axis. The zoom-in view shows the heme $c$ binding domains (D1 and D2) of QcrC. The heme groups $\left(b_{\mathrm{H}}, b_{\mathrm{L}}, c_{\mathrm{D} 1}\right.$, and $\left.\mathrm{C}_{\mathrm{D} 2}\right)$ and [2Fe-2S] clusters are shown as spheres and menaquinone/menaquinol (MK) are shown as sticks. The regions of ridge roof, ceiling junction, and base plate of $\mathrm{ClII}$ dimer are marked with dashed ellipses. (B) Overall structure of CIV (left) and the spatial location (right) of prosthetic groups. CtaC, CtaD, CtaE, CtaF, Ctal and CtaJ are colored in magenta, dark green, yellowish brown, cyan, brown and violet, respectively. Prosthetic groups are shown as spheres. 


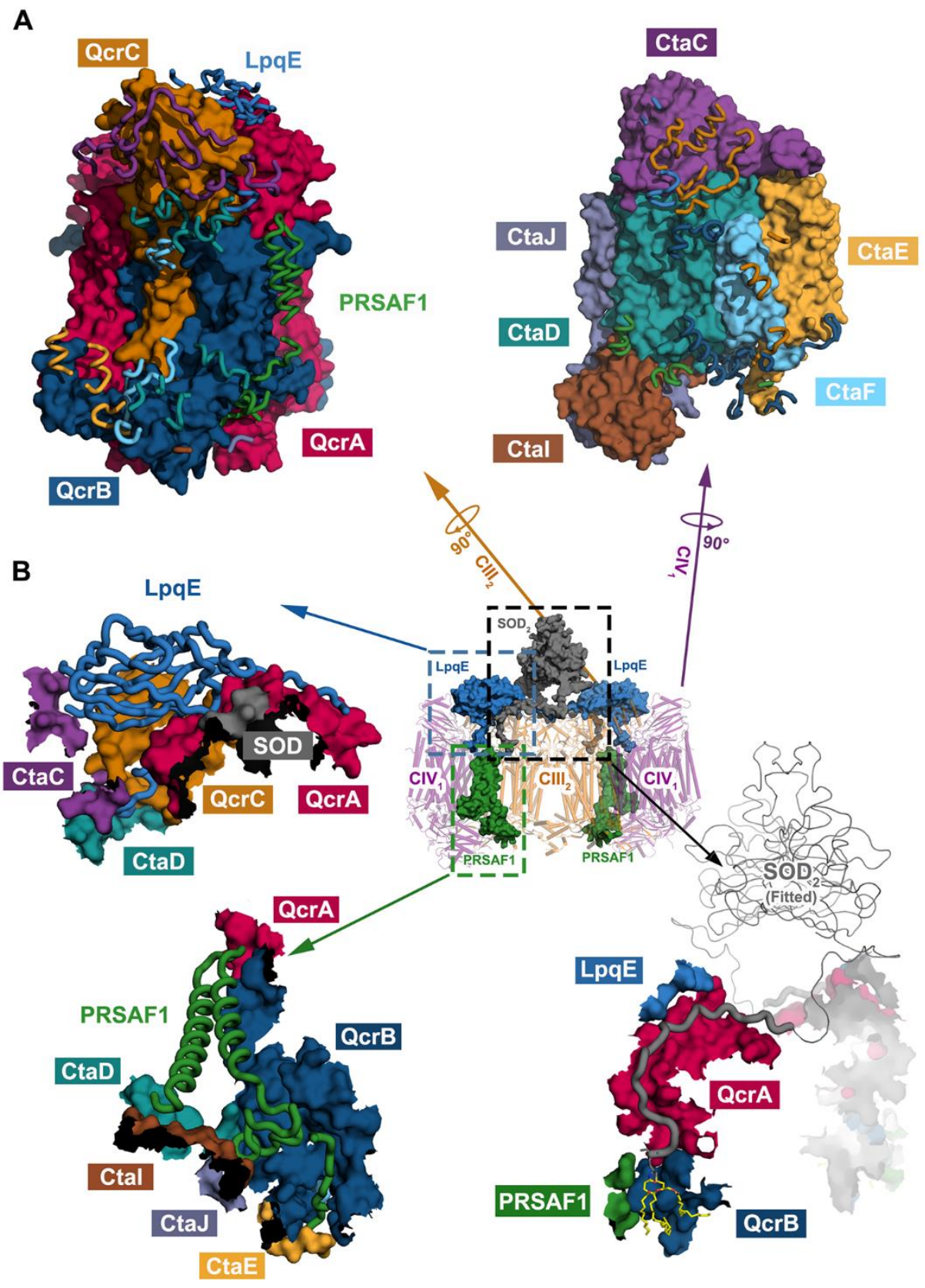

Fig. 3. Interaction between CIII and CIV and roles of association subunits. (A) A section profile of the interaction interface between CIII and CIV. The left/right images show the surface of CIII/CIV and the bound structural segments from CIV/CIII subunits and the association subunits, LpqE and PRSAF1. (B) Interactions between the association subunits (PRSAF1, LpqE and SOD) and the subunits of CIII and CIV. The corresponding subunits of $\mathrm{CIII}$ and CIV are shown in surface representation and colored as indicated. The association subunits are shown in ribbon and colored as indicated. The catalytic domain of SOD was fitted in map and shown as a dimer $\left(\mathrm{SOD}_{2}\right)$. The lipid modification of SOD is shown in yellow sticks (see Fig. 4E for detail). 
A
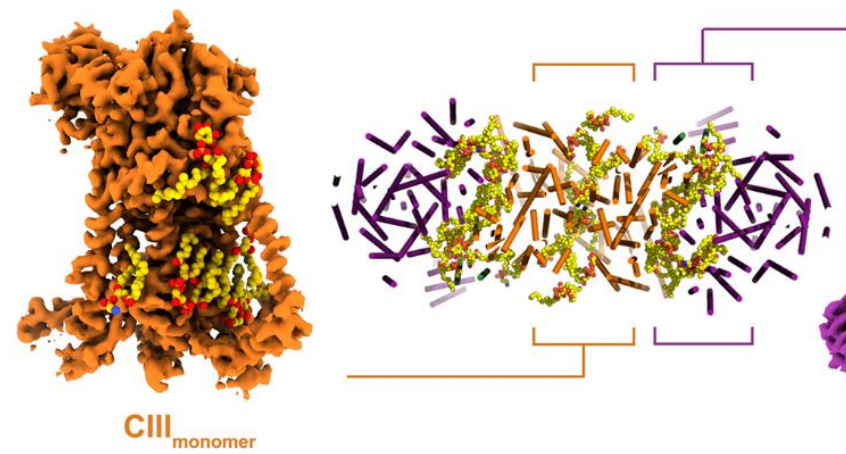

B

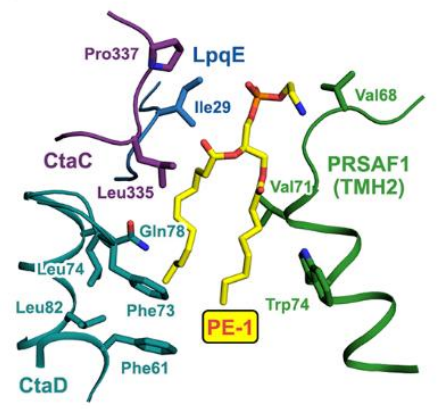

D

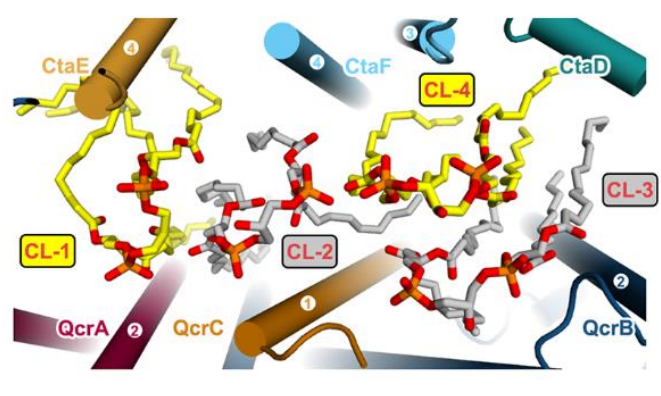

C

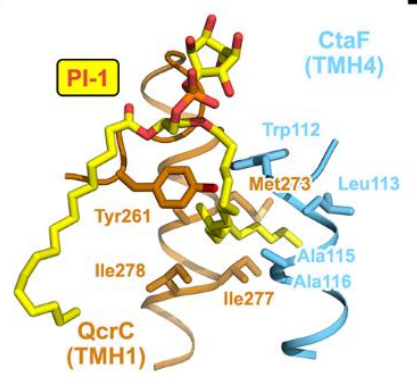

E

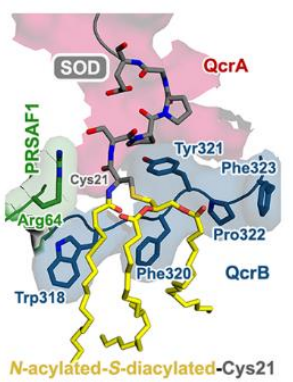

$\mathbf{F}$

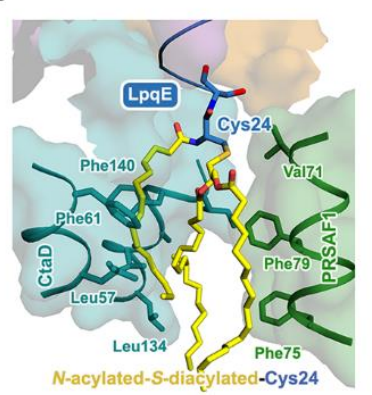

Fig. 4. Important roles of phospholipids in the stability and assembly of the supercomplex. (A) The distribution of phospholipids in the membrane region (middle, view from periplasmic side) at the junction between the Clll monomers (left) and the interfaces between $\mathrm{Clll}$ and CIV (right). (B) A PE molecule mediates the interaction between PRSAF1 (TMH2) and CIV (CtaC and CtaD). (C) A PI molecule binds to the interface between QcrCTMH1 and CtaTMH4. (D) Four CL molecules are bound in the groove between the TM regions of $\mathrm{Clll}$ and CIV (E) The N-terminal lipid modification of SOD at Cys21 binds to QcrB via hydrophobic interactions. (F) The N-terminal lipid modification of LpqE at Cys24 mediates the interactions between CtaD and PRSAF1. 
A

B
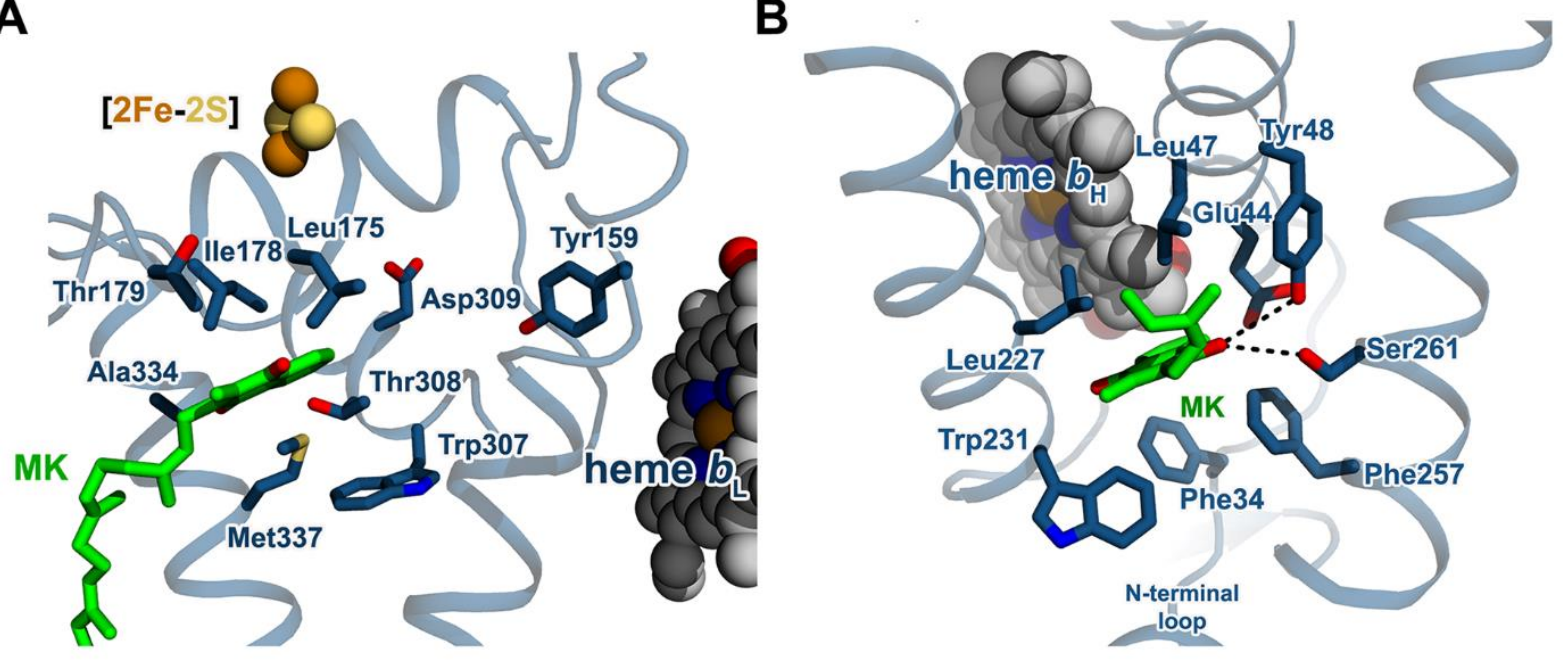

C

Mycobacterium smegmatis mc $51(\mathrm{QcrB})$
Mycobacterium tuberculosis H37Rv
Mycobacterium leprae TN
Nocardia lijiangensis NBRC 108240
Rhodococcus tukisamuensis NBRC 100609
Homo sapiens

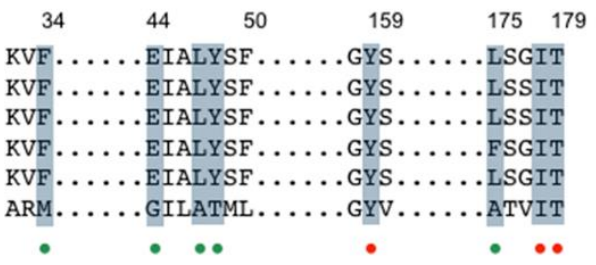

Mycobacterium smegmatis $\mathrm{mc}^{2} 51$ (QcrB) Mycobacterium tuberculosis H37Rv Mycobacterium leprae TN

Nocardia lijiangensis NBRC 108240 $\begin{array}{llllllll}224 & 234 & 256 & 262 & 301 & 310 & 332 & 340\end{array}$

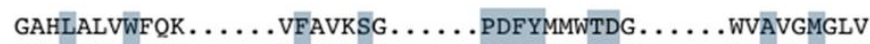
GLHLALVWFQK. . . . . V.VFAFKSG . . . . . PDFYMMWTEG . . . . . WVAVIMGLV GLHLALVWFQK . . . . V.VFAFKSG . . . . PDFYMMWTEG . . . . WVAVIMALV AAHVALVWYQK......VFAADQG ..... PDFYMMWTDG. . . . . WVALIMGLV Rhodococcus tukisamuensis NBRC 100609 GAHLALVWYQK......VFFVKSG......PDIYMMWTDG......AVALIMGLV Homo sapiens

Fig. 5. Structures of the menaquinone $(\mathrm{MK}) /$ menaquinol $\left(\mathrm{MKH}_{2}\right)$ binding sites in $M$. smegmatis CIII. (A) The $\mathrm{Q}_{P}$ binding site. (B) The $\mathrm{Q}_{\mathrm{N}}$ binding site. The residues potentially involved in the binding of $\mathrm{MK} / \mathrm{MKH}_{2}$ are shown with side chains in a stick representation. The MK molecules are colored in green. The heme and [2Fe-2S] groups are shown in spheres and labeled accordingly. (C) Sequence alignment for the $Q_{p}$ site and $Q_{N}$ site in QcrB with other Actinobacteria and Homo sapiens ETC systems. The red and green dots indicate whether Homo sapiens shares a common conservative site with Actinobacteria (red) or not (green). 
A

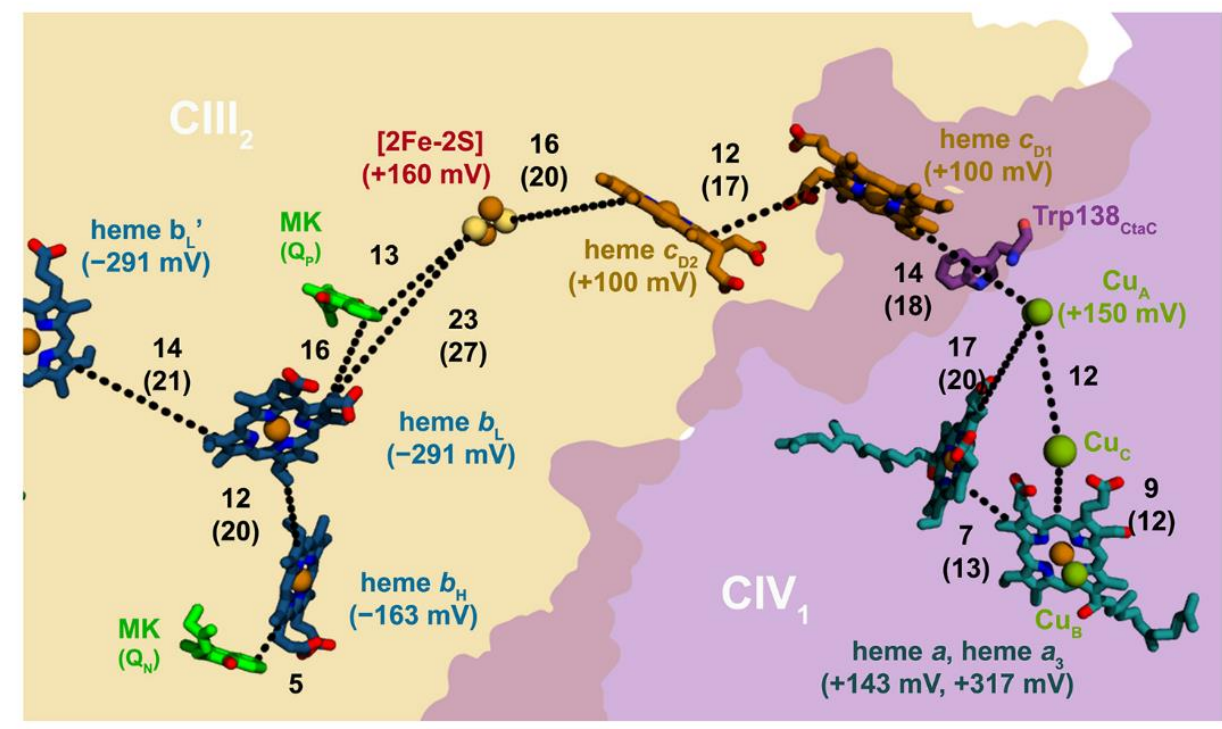

B

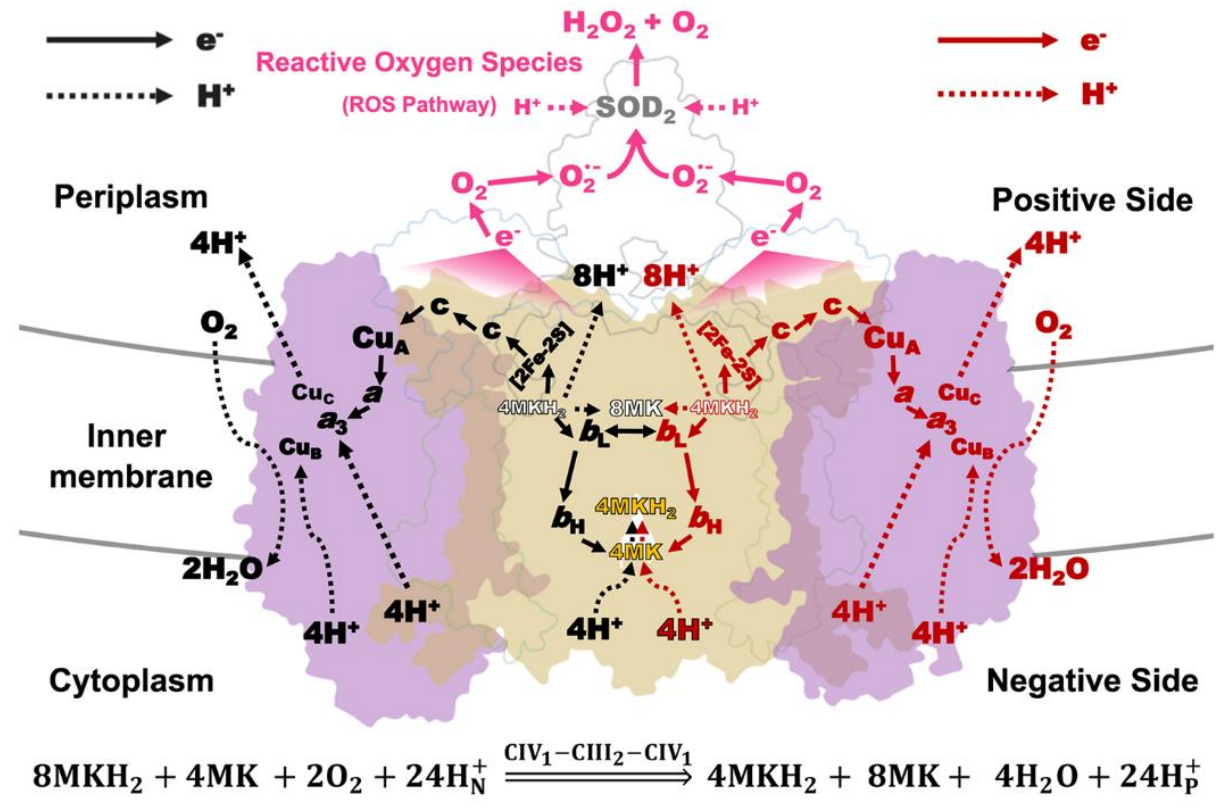

Fig. 6. The complete electron transfer pathway in the $b c c-a a_{3}$ type respiratory CIII/CIV supercomplex. (A) The prosthetic groups in the supercomplex are shown in sticks or spheres and labeled accordingly with the corresponding midpoint potentials shown in brackets. These values are based on the measurement of that of the bcc-aa $a_{3}$ type supercomplex from $C$. glutamicum (10). The edge-to-edge distances between adjacent prosthetic groups are shown in black dashed lines with the numbers in the brackets representing the centerto-center distances. (B) A schematic diagram showing the entire electron transfer pathway from CIII to CIV and the relevant proton translocations in Clll and CIV. The potential role of associated SOD for the clearance of ROS is also proposed. 

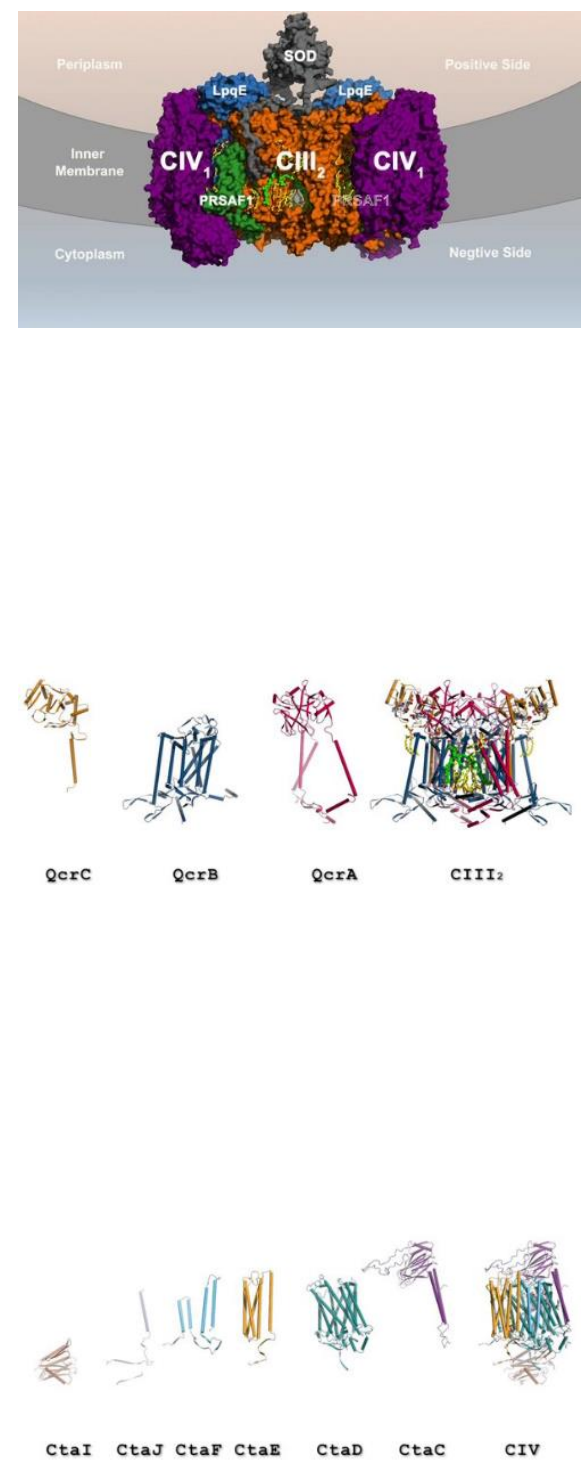

Movie 1. The overall architecture of the supercomplex. Cartoon representation of the supercomplex. The menaquinone/menaquinol (MK) is presented as bright green colored solid spheres and the phospholipids as yellow sticks.

Movie 2. The composition and structure of Clll dimer. Cartoon representation of the complex III. The menaquinone/menaquinol (MK) is presented as bright green colored solid spheres and the phospholipids as yellow sticks.

Movie 3. The composition and structure of CIV. Cartoon representation of the complex IV. The phospholipids are shown as vellow sticks. 